\title{
Effects of probiotic BaSC06 on intestinal digestion and absorption function, antioxidant capacity and macrophage polarization and microbiota composition in fattening pigs
}

\section{Xuefang Cao}

Zhejiang University

\section{Li Tang}

Zhejiang University

\section{Zihan Zeng}

Zhejiang University

\section{Baikui Wang}

Zhejiang University

Yuanhao Zhou

Zhejiang University

Qi Wang

Zhejiang University

Peng Zou

Zhejiang University

Weifen Li ( $\nabla$ wfli@zju.edu.cn )

Zhejiang University

\section{Research}

Keywords: Bacillus amyloliquefaciens SC06, antibiotics, fattening pigs, intestine, growth perfor-mance, antioxidant capacity, immune function, polarization, microbiota

Posted Date: May 4th, 2020

DOl: https://doi.org/10.21203/rs.3.rs-25137/v1

License: (c) (i) This work is licensed under a Creative Commons Attribution 4.0 International License. Read Full License 
Version of Record: A version of this preprint was published at Frontiers in Veterinary Science on November 6th, 2020. See the published version at https://doi.org/10.3389/fvets.2020.570593. 


\section{Abstract}

Background: The previous study in our team found that supplementation of probiotic Bacillus amyloliquefaciens SCO6 (BaSC06) as an alternative to antibiotics (Kitasamycin) can effectively reduce the diarrhea rate and promote the growth performance of weaned piglets. We speculate that BaSC06 may become a better substitute for antibiotics for fattening pigs. Hence, the objective of the present study is to evaluate the effects of BaSC06 substitute antibiotics on growth performance, digestive functions, antioxidant capacity, macrophage polarization and intestinal microbiota of fattening pigs.

Methods: A total of 117 fattening pigs with similar weight and genetic basis were selected and divided into three groups: Anti group, fed with basal diet supplemented with $40 \mathrm{~g} / \mathrm{t}$ Kitasamycin; Anti + Ba group, fed with $1 \times 10^{8} \mathrm{CFU} / \mathrm{kg}$ BaSC06 and $20 \mathrm{~g} / \mathrm{t} \mathrm{Kitasamycin;} \mathrm{Ba} \mathrm{group,} \mathrm{fed} \mathrm{with} 1 \times 10^{8} \mathrm{CFU} / \mathrm{kg} \mathrm{BaSC06}$ replaced Kitasamycin. Each treatment had three replications of 13 pigs per pen.

Results: It indicated that BaSC06 replacement significantly improved ADFI and ADG in fattening pigs. It also improved intestinal digestion and absorption function with the increased activities of intestinal digestive enzymes and higher expression of glucose transporters SGLT1 and small peptide transporters PEPT1, maintained intestinal integrity significantly. Besides, supplementation with BaSC06 enhanced intestinal and body antioxidant capacity by activating Nrf2/ Keap1 antioxidant signaling pathway. In addition, BaSC06 as a substitute could alleviate intestinal inflammation by inhibiting the production of pro-inflammatory cytokines and chemokine and simultaneously increase the expression of M1 and M2 macrophage marker proteins in the intestinal mucosa. The transcription factor STAT3 regulated M2 polarization, which maintained immune homeostasis and reduced inflammation. What's more, BaSC06 could improve the composition of the microbiota as well by increasing the proportion of Firmicutes, and reducing the proportion of Bacteroidetes and Proteobacteria.

Conclusions: Taken together, our findings indicated that diet supplementation with BaSC06 instead of antibiotics increased the growth performance of piglets by improving the digestion and absorption of nutrients, intestinal microbiota composition and mucosal structure, antioxidative capacity and immune functions (including increasing M1and M2 polarization of macrophage).

\section{Background}

In recent years, the serious drug resistance caused by antibiotics abusing in the animal husbandry industry has been widely concerned by the society. At present, China produces about 200,000 tons of antibiotics every year, accounting for about $50 \%$ of the world's consumption, of which about 97,000 tons are used for husbandry industry. Large doses of antibiotics consumption develop resistance, which makes the prevention and treatment effects of antibiotic worse and worse. People have to increase the dosage of antibiotics that cause a vicious circle. $30 \%-90 \%$ of antibiotics will be discharged with the animal feces, which causing certain harm to the environment. Additionally, antibiotics are rarely absorbed by organs but meat, eggs and milk, resulting in serious antibiotic residues of livestock products, posing a 
threat to people's food safety. Recently, the Ministry of Agriculture of People's Republic of China (PRC) issued the feed 'anti-drug prohibition' (Announcement No. 194), which explicitly requires that the use of feed additives containing growth-promoting antibiotics shall be strictly prohibited from January 1 in 2020. After December 31, 2020, the antibiotics feed additive will disappear in the feed industry. Therefore, the substitution of antibiotics, such as probiotics, prebiotics, plant extracts and organic acids, will be widely used in the animal husbandry industry. Different from the traditional chemical drugs that block or interfere with the metabolism of pathogenic bacteria, probiotics are promising and potential supplements, which can improve intestinal health in a variety of ways. Probiotics provide a potential alternative strategy for feeding antibiotics. The most commonly used probiotics in animal production process are Lactobacillus and Bacillus [1-4])

Bacillus amyloliquefaciens is a probiotic strain that produces several extracellular enzymes to augment digestibility and absorption of nutrients in addition to the improvement of overall intestinal immune function [5-7]. Bacillus amyloliquefaciens is preferred to be a feed supplement due to its higher resistance to harsh environments [8]. Besides, our previous studies based on piglets have demonstrated that probiotic $\mathrm{BaSC} 06$ significantly increase growth performance and antioxidant status by improving the digestive and absorb enzyme activities, antioxidant capacity, and intestinal autophagy; enhancing the intestinal integrity by improving intestinal mucosa structure, tight junctions and regulating the population of intestinal microbiota; increasing immune function by activating the TLRs signaling pathway $[9,10,11]$. Although BaSC06 has benefits mentioned before for production performance, comparative experiments on antibiotics are still scarce. To get a comprehensive assessment of the intestinal physiological and biochemical situation, this research was conducted to evaluate the effects of BaSC06 on the intestinal enzymatic activities for digestion and absorption, antioxidant capacity, immune function and macrophage polarization in fattening pigs.

\section{Materials And Methods}

\subsection{Animals and Diets}

A total of 117 fattening pigs (Duroc $\times$ Landrace $\times$ Yorkshire) with a similar initial weight of $53 \mathrm{~kg}$ and a similar genetic basis were selected and randomly divided into 3 groups with 13 replicates per pen. Fattening pigs in the Anti group were fed with the normal diet containing $40 \mathrm{~g} / \mathrm{t}$ Kitasamycin. Fattening pigs in Ba group were fed the basal diet supplemented with $1 \times 10^{8} \mathrm{CFU} / \mathrm{kg}$ BaSC06 without any antibiotics. Fattening pigs in the Anti+Ba group were fed with the basal diet containing $20 \mathrm{~g} / \mathrm{t} \mathrm{Kitasamycin}$ and $1 \times 10^{8} \mathrm{CFU} / \mathrm{kg} \mathrm{BaSC06}$. All the fattening pigs were fed ad libitum to basal feed and water. The experiment period lasted 7 days, while the formal experiment period lasted 41 days, followed by sample collection and data analysis. All the experimental procedures were approved by and performed according to the guidelines of the Animal Care and Use Committee of Zhejiang University. The corn-soybean mealbased diet was supplemented with minerals and vitamins regarding to the nutritional requirements of NRC (2012). The basal diet composition and nutrition level are shown in the table below. 


\begin{tabular}{lclc}
\hline \multicolumn{3}{c}{ TABLE 1 | Ingredient and chemical composition of the basal diet (as-fed basis). } \\
\hline Ingredient & Content (\%) & Nutition levels & Content (\%) \\
\hline Corn & 60.21 & DE (Mcal/kg) & 3.41 \\
Soybean meal & 24.55 & CP (\%) & 17.22 \\
Wheat bran & 4.5 & Lys (\%) & 0.84 \\
Soybean oil & 3.8 & Met (\%) & 0.27 \\
Corn starch & 2.05 & Thr (\%) & 0.63 \\
Premix a & 3 & Ca (\%) & 0.73 \\
Salt & 0.09 & Available P (\%) & 0.27 \\
CaHPO4 & 0.9 & & \\
Limestone & 0.9 & & \\
Total & 100 & & \\
\hline
\end{tabular}

aPremix supplied per kg: $100 \mathrm{mg}$ of $\mathrm{Fe}$ (FeSO4), $10 \mathrm{mg}$ of $\mathrm{Cu}$ (CuSO4), $100 \mathrm{mg}$ of $\mathrm{Zn}$ (ZnO), 10mg of Mn (MnO), $3086 \mathrm{IU}$ of vitamin A, $386 \mathrm{IU}$ of vitamin D3, $15.4 \mathrm{IU}$ of vitamin E, 3.9mg of vitamin B2, $2.3 \mathrm{mg}$ of vitamin $\mathrm{K} 3,15.4 \mathrm{ug}$ of B12, 15.4 ug of vitamin B5, 23 ug of vitamin B3.

\subsection{Bacterial Strain and Kitasamycin}

Bacillus amyloliquefaciens cells (CCTCC No: M 2012280) $\left(1 \times 10^{8} \mathrm{CFU} / \mathrm{g}\right)$ were prepared by the Laboratory of Microbiology, Institute of Feed Sciences, Zhejiang University, China P.R. Starch was used to dilute BaSC06 and the same amount of starch was also added to each group to compensate for the difference in nutrient composition of the diets. Kitasamycin was obtained from Tongyi feed agriculture and animal husbandry Co., Ltd. (Qingdao, PRC).

\subsection{Sample Collection}

At the end of the experiment, fattening pigs were randomly selected from each group $(n=6)$ for sample collecting. Pigs were slaughtered after 24 hours of fasting. Middle and posterior ileum were removed and rinsed with sterile saline, then the ileum mucosa were scraped off. Samples of ileum tissue were stored in $10 \%$ formaldehyde solution for histological observation. Intestinal mucosa and contents samples of duodenum, jejunum, ileum and cecum were also collected. All samples were immediately frozen in liquid nitrogen and then stored at $-80^{\circ} \mathrm{C}$ for further analysis.

\subsection{Growth Performance Assay}

Weight gain (g) $\llbracket$ (weight at the end of the experiment $(\mathrm{kg})$ - weight at the beginning of the experiment $(\mathrm{kg})) / 1000$

Average daily gain (ADG) (g)凶Weight gain per pig (g)/days(d) 
Feed/Gain (F/G)『Feed intake per pig throughout the trial period (g)/ Weight gain per pig (g)

\subsection{Hematoxylin and Eosin (H\&E) Staining}

The prepared paraffin sections ( $5 \mu \mathrm{m}$ thick) were subjected with H\&E staining and observed by a light microscope (Nikon Eclipse 80i, Tokyo, Japan). The height of the ileum villi and crypt, the thickness of the mucosa were measured with a micrometer, and the height of the villi/the depth of the crypt $(\mathrm{V} / \mathrm{C})$ was calculated.

\subsection{Biochemical Analyses}

Duodenal contents digestive enzymes $₫$ weigh about $0.5 \mathrm{~g}$ of duodenal contents, add $0.9 \%$ normal saline of appropriate volume according to the mass volume ratio of 1:9, and keep it on ice with an electric homogenizer to fully homogenize. The homogenate was centrifuged for $15 \mathrm{~min}$ at $4{ }^{\circ} \mathrm{C}$ and $3500 \mathrm{r} / \mathrm{min}$. Then the protein concentration was determined. The activities of chymotrypsin, amylase, trypsin, and lipase were determined by kit.

Jejunum antioxidase activity: take jejunum tissue samples, the method is the same as above. The kit determined the total antioxidant capacity (T-AOC), Methane dicarboxylic aldehyde (MDA), Glutathione (GSH), Catalase (CAT), Total Superoxide Dismutase (T-SOD) and Glutathione peroxidase (GSH-Px) activity.

Intestinal mucosa disaccharidase and absorptive enzyme: take appropriate jejuna mucosa samples; the method is the same as above. $y$-glutamyl transpeptidase ( $(y-G T)$ kit, Alkaline phosphatase (AKPase) kit and Sodium potassium ATPase $\left(\mathrm{Na}^{+}, \mathrm{K}^{+}\right.$-ATPase) kit were utilized to assay the activity of absorption enzymes. All the kits above were purchased from Nanjing Jiancheng Bioengineering Research Institute, and the operation method was following the instructions of the kits.

\subsection{RNA Extraction and Real-time Quantitative PCR}

Total RNA isolated from intestinal mucosa (RNAiso plus, TaKaRa, Dalian, PRC) was reverse transcribed using a Prime Script II 1st Strand cDNA Synthesis Kit (TaKaRa). Quantitative and qualitative analyses of isolated RNA were assessed from the ratio of absorbance at 260 and $280 \mathrm{~nm}$ by NanoDrop spectrophotometer (Thermo Scientific, Wilmington, DE, USA) and agarose gel electrophoresis (Sangon Biotech, Shanghai, China). Complementary DNA (cDNA) was synthesized from I $\mu \mathrm{g}$ of total RNA using MMLV reverse transcriptase (TaKaRa). RT-qPCR was performed according to a previous study [9] using the Premix Ex TaqTM with SYBR Green (TaKaRa), and the ABI 7500 Fast Real-Time PCR system (Applied Biosystems, Carlsbad, CA, USA). The thermocycling protocol lasted for $30 \mathrm{~s}$ at $95^{\circ} \mathrm{C}$, followed by 40 cycles of $5 \mathrm{~s}$ denaturation at $95^{\circ} \mathrm{C}, 34 \mathrm{~s}$ annealing/extension at $60^{\circ} \mathrm{C}$, and then a final melting curve analysis to monitor the purity of the PCR product. Primer sequences were designed and selected by Primer 5.0 and Oligo 7.0 software and the sequences are presented in Supplementary Table S1. The 2- 
$\triangle \Delta \mathrm{Ct}$ method was used to estimate mRNA abundance. Relative gene expression levels were normalized to those of the eukaryotic reference gene glyceraldehyde-3-phosphate dehydrogenase (GAPDH).

\subsection{Western Blotting}

Tissue lysates were prepared using Cell Lysis Buffer for Western and IP (Biotime Biotechnology, Xiamen, Fujian, PRC). Protein concentrations were detected using Enhanced BCA Protein Assay Kit (Biotime Biotechnology). Equal amounts of proteins from each sample were subjected to SDS-PAGE followed by transfer of proteins to nitrocellulose membranes. Membranes were blocked with a non-protein blocking solution (Sangon Biotech) and incubated with a primary antibody overnight at $4{ }^{\circ} \mathrm{C}$. After washing with TBST, membranes were incubated with a secondary antibody linked to Horseradish peroxidase (HRP). The blots were then developed with an ECL detection system as per the manufacturer's instructions. Rabbit anti-SGLT1 (ab14686), anti-PEPT1 (ab203043), anti-P47 ${ }^{\text {phox }}$ (ab74095), anti-Nrf2 (ab62352), antiKeap1 (ab139729), anti-iNOS (ab178945), anti-MR (ab229987), anti-p-IRF3 (Ser396)(ab138449), antiIRF3 (ab25950), monoclonal antibodies were obtained from Abcam. Rabbit anti-Arg (93668S), antipSTAT1(Tyr701)(9167S), anti-STAT1 (9172T), anti-STAT6 (5397S), anti-STAT3 (30835S) monoclonal antibodies were obtained from CST (Shanghai, PRC). Mouse anti- $\beta$-actin monoclonal antibody was obtained from Biotime Biotechnology.

\subsection{Cytokine Assay}

Levels of Tumor Necrosis Factor-a (TNF-a), Interleukin-6 (IL-6), IL-8, IL-4, and Monocyte Chemotactic Protein 1 (MCP1) in the ileum mucosa supernatants were quantified using ELISA kits (eBioscience, Santa Clara, CA, USA) as per manufacturer's instruction.

\subsection{DNA Extraction, Pyrosequencing and Bioinformatics Analysis}

The DNA Isolation Kit (Tiangen, Beijing, China) was used for cecum DNA extraction and the quality of the extracted DNA was checked by agarose gel electrophoresis and spectrophotometric analysis. All of the genomic DNA samples were stored at $-80^{\circ} \mathrm{C}$ for further experiments. 16 s rRNA PCR amplification and 454 pyrosequencing were performed according to Qin et al. (2018) [12]. Sequences obtained through 454 pyrosequencing were then filtered by QIIME software (QIIME version 1.9.1) with default parameters [13]. The operational taxonomic unit (OTU) clustering pipeline UPARSE was used to select OTU at $97 \%$ similarity. Alpha diversity and beta diversity between the samples were also analyzed by QIIME software. The final taxonomic assignment was based on the consensus identification for each OTU.

\subsection{Statistical Analysis}

The data in the present study were analyzed by one-way ANOVA using the IBM SPSS 16.0. The values of $\mathrm{P}<0.05$ or 0.01 were considered a statistically significant difference.

Table 2 Analysis of growth performance of fattening pigs $(n=13)$ 


\begin{tabular}{lccc}
\hline Items & Anti group & Anti+Ba group & Ba group \\
\hline Initial body weight $(\mathrm{kg})$ & $55.19 \pm 3.22$ & $54.97 \pm 1.61$ & $52.38 \pm 2.93$ \\
Final body weight $(\mathrm{kg})$ & $88.19 \pm 2.65$ & $91.77 \pm 3.22$ & $88.98 \pm 2.95$ \\
Average daily feed intake(g/d) & $2391.11 \pm 144.22$ & $2505.37 \pm 279.90$ & $2440.59 \pm 184.45$ \\
Average daily gain $(\mathrm{g})$ & $804.88 \pm 1.32^{\mathrm{a}}$ & $897.56 \pm 3.35^{\mathrm{b}}$ & $892.68 \pm 2.91^{\mathrm{b}}$ \\
Feed/Gain & $2.97 \pm 0.45$ & $2.81 \pm 0.24$ & $2.72 \pm 0.12$ \\
\hline
\end{tabular}

Data were expressed as mean \pm SD. In the same row, values with different small letter superscripts mean significant difference $(P<0.05)$, and with the same or no letter superscripts mean no significant difference $(P<0.05)$. The following tables and figures are also consistent. Anti group=basal diet with 40g/t kitasamycin; Anti+Ba group=basal diet containing 20g/t kitasamycin and $1 \times 10^{8} \mathrm{CFU} / \mathrm{kg}$ BaSC06; Ba group = basal diet containing $1 \times 10^{8} \mathrm{CFU} / \mathrm{kg} \mathrm{BaSC} 06$.

\section{Results}

\subsection{Effects of BaSC06 on Growth Performance of Fattening Pigs}

Five indexes (Initial body weight, Final body weight, Average daily feed intake(ADFI), Average daily gain(ADG) and Feed/Gain(F/G)) for the growth performance of fattening pigs were measured. Compared with the Anti group, $A D G$ in the Ba group significantly increased $(P<0.05)$, and $A D F I$ in the Anti + Ba group and $\mathrm{Ba}$ group showed an increasing trend, which indicated that BaSC06 administration instead of antibiotics could improve the growth performance of fattening pigs.

\subsection{Effects of BaSC06 on Morphology of Ileum Mucosa in Fattening Pigs}

The visual effects on the morphology of ileum mucosa in fattening pigs could be directly observed in Fig.1. Compared to ordinary Kitasamycin treatment (Anti group), the ileum villus of the fattening pigs in Anti+Ba and Ba group developed better with more morphological integrity (Fig. 1) and higher villus height $(P<0.05)$ (Table. 3). However, the crypt depth in the Anti+Ba group and Ba group showed no significant changes compare to the Anti group ( $P>0.05)$, while $V / C$ showed a certain increasing trend.

Table 3 Analysis of BaSC06 on intestinal morphology of fattening pigs $(n=6)$

\begin{tabular}{lccc}
\hline Items & Anti group & Anti+Ba group & Ba group \\
\hline villus height $(\mu \mathrm{m})$ & $253.83 \pm 2.77^{\mathrm{a}}$ & $281.26 \pm 2.44^{\mathrm{b}}$ & $286.50 \pm 1.94^{\mathrm{b}}$ \\
crypt depth $(\mu \mathrm{m})$ & $122.08 \pm 4.37$ & $132.46 \pm 3.23$ & $134.39 \pm 23.38$ \\
villus height: crypt depth & $2.08 \pm 0.03$ & $2.12 \pm 0.02$ & $2.13 \pm 0.04$ \\
\hline
\end{tabular}

\subsection{Effects of BaSC06 on Digestion and Absorption Functions of Fattening Pigs}

\subsubsection{Effects of BaSC06 on Intestinal Digestive Enzyme Activities}


As shown in Table 4, compare to Anti group, the activities of trypsin, chymotrypsin and amylase in duodenal contents and trypsin and amylase in jejuna contents were significantly increased in both Anti+Ba group and Ba group $(P<0.05)$, whereas the chymotrypsin and lipase activities in the jejuna contents in BaSC06 supplementary groups (Anti+ Ba group and Ba group) were significantly decreased ( $P$ $<0.05)$, and there was no significant difference for the lipase in the duodenal content among three groups $(P>0.05)$.

Table 4 Analysis of the digestive enzyme activities in intestinal contents of fattening pigs

\begin{tabular}{lccc}
\hline Items & Anti group & Anti+Ba group & Ba group \\
\hline Duodenum & & & \\
Trypsin(U/mgprot) & $261.60 \pm 25.61^{\mathrm{b}}$ & $507.72 \pm 3.91^{\mathrm{a}}$ & $465.38 \pm 26.99^{\mathrm{a}}$ \\
Chymotrypsin(U/gprot) & $3594.01 \pm 384.10^{\mathrm{b}}$ & $6074.17 \pm 597.89^{\mathrm{a}}$ & $2718.31 \pm 128.35^{\mathrm{b}}$ \\
Amylase(U/mgprot) & $519.10 \pm 25.81^{\mathrm{b}}$ & $746.66 \pm 46.52^{\mathrm{a}}$ & $861.53 \pm 26.28^{\mathrm{a}}$ \\
Lipase(U/gprot) & $22.92 \pm 2.08$ & $14.37 \pm 0.04$ & $14.56 \pm 5.40$ \\
\hline Jejunum & & & \\
Trypsin(U/mgprot) & $576.80 \pm 63.71^{\mathrm{b}}$ & $1022.91 \pm 39.52^{\mathrm{a}}$ & $580.42 \pm 33.62^{\mathrm{b}}$ \\
Chymotrypsin(U/gprot) & $6334.65 \pm 580.79^{\mathrm{a}}$ & $4155.19 \pm 369.86^{\mathrm{b}}$ & $2154.20 \pm 223.70^{\mathrm{c}}$ \\
Amylase(U/mgprot) & $767.73 \pm 20.49^{\mathrm{b}}$ & $1221.74 \pm 19.75^{\mathrm{a}}$ & $744.73 \pm 24.71^{\mathrm{b}}$ \\
Lipase(U/gprot) & $159.20 \pm 7.45^{\mathrm{a}}$ & $60.30 \pm 4.55^{\mathrm{b}}$ & $29.81 \pm 3.51^{\mathrm{c}}$ \\
\hline
\end{tabular}

Data were analyzed by one-way ANOVA Tukey test ( $\mathrm{n}=8$ in each group). Different letters $(\mathrm{a}, \mathrm{b}, \mathrm{c})$ in each parameter represent significant $(p<0.05)$.

\subsubsection{Effects of BaSC06 on Jejuna Mucosa Disaccharide Enzyme Activity}

As shown in Table 5, BaSC06 can enhance mucosal disaccharide enzyme activity in jejuna. Compared with the Anti group, the sucrase and lactase activities in the Ba group significantly increased by $66.4 \%$ ( $P$ $<0.05)$ and $100 \%(P<0.05)$, but there was no significant effect on three disaccharide enzymes (sucrase, maltase, lactase) in Anti+Ba group. Compared with the Anti+Ba group, the sucrase and lactase activities in the Ba group were significantly increased by $39.8 \%(P<0.05)$ and $105.7 \%(P<0.05)$, but the maltase activity decreased insignificantly $(P>0.05)$. So, it could be concluded that BaSC06 could improve the digestion and absorption of carbohydrates by increasing jejuna mucosal disaccharide enzyme activity in fattening pigs, and the effects of using probiotics alone was better than using both of probiotics and antibiotics.

Table 5 Analysis of the disaccharidase activities in jejunum mucosa of fattening pigs 


\begin{tabular}{lccc}
\hline Items & Anti group & Anti+Ba group & Ba group \\
\hline Sucrase(U/mgprot) & $86.65 \pm 1.52^{\mathrm{b}}$ & $103.14 \pm 10.23^{\mathrm{b}}$ & $144.16 \pm 3.14^{\mathrm{a}}$ \\
Maltase(U/mgprot) & $209.94 \pm 8.53$ & $196.47 \pm 22.69$ & $179.89 \pm 11.78$ \\
Lactase(U/mgprot) & $1.09 \pm 0.10^{\mathrm{b}}$ & $1.06 \pm 0.19^{\mathrm{b}}$ & $2.18 \pm 0.12^{\mathrm{a}}$ \\
\hline
\end{tabular}

Data were analyzed by one-way ANOVA Tukey test ( $\mathrm{n}=8$ in each group). Different letters (a, b, $c)$ in each parameter represent significant $(p<0.05)$.

\subsubsection{Effects of BaSC06 on Enzyme Activity Related to Absorption in Jejuna Mucosa of the Fattening Pigs}

Compared with Anti group, half and total replacing Kitasamycin with BaSC06 could dramatically sparked the activity of AKPase and $\mathrm{Na}^{+}, \mathrm{K}^{+}$-ATPase in jejuna mucosa $(\mathrm{P}<0.05)$ (Table 6), increasing by $47.3 \%$ for AKPase in Anti+Ba group and by $76.6 \%$ for $\mathrm{Na}^{+}, \mathrm{K}^{+}$-ATPase in the Ba group, respectively (Table 6 ). Feed supplemented with only BaSC06 did dramatically increased $\mathrm{Y}-\mathrm{GT}$ activity $(P<0.05)$, while no significant difference of $y-G T$ activity was found in half antibiotic replacing group $(P>0.05)$ (Table 6). These results indicated that the feed supplemented with BaSC06 instead of antibiotics can regulate the activity of intestinal mucosa-associated absorption enzymes in fattening pigs.

Table 6 Analysis of the absorptive enzyme activities in jejunum mucosa of fattening pigs

\begin{tabular}{lccc}
\hline Items & Anti group & Anti+Ba group & Ba group \\
\hline$\gamma$-Glutamytransferase(U/gprot) & $40.58 \pm 1.15^{\mathrm{a}}$ & $37.78 \pm 2.94^{\mathrm{a}}$ & $25.41 \pm 2.48^{\mathrm{b}}$ \\
AKPase(Kingunit /gprot) & $748.25 \pm 6.56^{\mathrm{b}}$ & $1102.10 \pm 57.66^{\mathrm{a}}$ & $679.67 \pm 19.33^{\mathrm{b}}$ \\
$\mathrm{Na}^{+}, \mathrm{K}^{+}$-ATPase(U/mgprot) & $3.50 \pm 0.23^{\mathrm{b}}$ & $5.17 \pm 0.23^{\mathrm{a}}$ & $6.18 \pm 0.50^{\mathrm{a}}$ \\
\hline
\end{tabular}

Data were analyzed by one-way ANOVA Tukey test ( $\mathrm{n}=8$ in each group). Different letters (a, b, $c)$ in each parameter represent significant $(p<0.05)$.

\subsubsection{Effects of BaSC06 on Transporter Expression}

Compared with the Anti group, administration of BaSC06 significantly increased the expression of the glucose transporter sodium-dependent glucose transporters 1(SGLT1) in the jejunum $(P<0.05)$, which were significantly up-regulated about 4 times in the Anti+Ba and Ba groups $(P<0.05)$ (Fig. 2A). However, there was no significant difference in the mRNA expression level of glucose transporter 2 (GLUT2) between Ba group and Anti + Ba group $(P>0.05)$ (Fig. 2A). The results of the expression of five amino acid transporters showed that BaSC06 could markedly regulate mRNA expression levels of the neutral amino acid transporter amino-acid transporter 2 (ASCT2), cationic amino acid transporter L-type amino 
acid transporter 1 (LAT1), and small peptide transporter 1 (PEPT1) in the jejuna mucosa. Compared with the Anti group, the expression of LAT1 mRNA decreased significantly in Ba group and Anti+Ba group ( $P$ $<0.05$ ) (Fig. 2B), A significant decreased in the mRNA expression level of ASCT1 and the increased in PEPT1 were observed in $\mathrm{Ba}$ group $(\mathrm{P}<0.05)$ (Fig. 2B). Neither the neutral amino acid transporter $\mathrm{B}^{0}$-type amino acid transporter $1\left(\mathrm{~B}^{0} \mathrm{AT} 1\right)$ nor the acidic amino acid transporter excitatory amino acid carrier 1 (EAAC1) was significantly changed in the three groups $(P>0.05)$ (Fig. 2B). We further verified the expression levels of SGLT1 and PEPT1 by Western blotting and obtained similar results. Compared with the Anti group, feed supplemented with $\mathrm{BaSC06}$ alone significantly increases the protein expression levels of $\mathrm{Na}^{+}$-dependent glucose transporter SGLT1 and small peptide transporter PEPT1 in the jejunum by $78.3 \%(P<0.05)$ and $177.4 \%$, respectively $(P<0.05)$ (Fig. $2 C)$. Therefore, it was concluded that BaSC06 instead of antibiotics could increase the expression of glucose transporters and small peptide transporters in the intestinal mucosa, and promote the absorption as well as transportation of glucose and small peptides in intestines.

\subsection{Effects of BaSC06 on Antioxidant Capacity}

\subsubsection{Effects of BaSC06 on Intestinal Antioxidant Enzyme Activities}

BaSC06 supplementary can reduce the MDA level in the intestine by $48.9 \%(P<0.05)$ and $42.0 \%(P=$ 0.061) for Anti+Ba and Ba group, respectively (Fig. 3B). At the same time, BaSC06 supplementary could significantly improve the intestinal T-AOC content $(P<0.05)$ (Fig. 3B), and compared to Anti group, CAT and GSH-Px activities increased by $18.7 \%$ and $25.4 \%$ in Anti + Ba group ( $>0.05)$ (Fig.3A) and increased by $45.4 \%(P<0.05)$ and $49.4 \%(P<0.05)$ in Ba group. There was no significant difference in T-SOD activity and GSH level between the Anti+Ba group and Ba group $(P>0.05)$ (Fig. $3 A$ ). The results above suggested that BaSC06 could enhance the intestinal antioxidant capacity by increasing the activity of antioxidant enzymes, moreover, the effect of BaSC06 supplementary alone was better than the combination of antibiotics and BaSC06.

\subsubsection{Effects of Replacing Kitasamycin with BaSC06 on Antioxidant-related Genes mRNA Expression in Jejunum of Fattening Pigs}

The expression of antioxidant-related genes is also an important indicator for determining the antioxidant capacity of the body. Antibiotics replaced by BaSC06 significantly increased the relative expression level of NAD (P)H: Quinone oxidoreductase 1(NQ01), and reduced the relative expression level of Hemeoxygenase-1 (HO-1). Compared with the control group, the mRNA expression of the NQ01 gene in the Anti+Ba group was significantly increased by $108.5 \%(\mathrm{P}<0.05)$ (Fig. $3 \mathrm{C}$ ), while the HO-1 gene mRNA expression in the Anti+Ba group and the Ba group was significantly decreased by $85.3 \%$ and $76.1 \%$ ( $P$ $<0.05$ ) (Fig. 3C). There was no significant difference in the expression of glutathione S-transferase (GST) mRNA among the three groups $(P>0.05)$ (Fig. 3C).

\subsubsection{Replacing Kitasamycin with BaSC06 Activated Nrf2/Keap1 Signaling Pathway in Jejuna of Fattening Pigs}

Page $11 / 35$ 
Compared with the Anti group, the relative expression of $\mathrm{P} 47^{\text {phox }}$ protein in the jejuna mucosa in the diet supplemented with half BaSC06 and half Kitasamycin was significantly reduced by $36.8 \%(P<0.05)$ (Fig.3D), while there was no significant difference between the Ba and Anti groups ( $P>0.05)$ (Fig.3D). The results indicated that BaSCO6 could reduce NADPH oxidase (NOX) activity by reducing the expression of $\mathrm{P} 47^{\text {phox }}$, preventing excessive production of reactive oxygen species (ROS) as well as reduce oxidative stress. Compared to Anti group, the phosphorylated Nuclear transcription factor-erythroid 2-related factor (Nrf2) proteins in the jejunum of the Anti+Ba group and Ba group were significantly up-regulated by 64.9\% $(P<0.05)$ and 74.3\% $(P<0.05)$, although the relative expression levels of Kelch-like epichlorohydrinassociated protein 1 (Keap1) and Nrf2 proteins did not significantly alter $(P>0.05)$, which implied that BaSC06 could improve the antioxidative capacity by activating the Nrf2 / Keap1 signaling pathway in jejuna mucosa of fattening pigs.

\subsection{Effects of BaSC06 on Immune Function}

\subsubsection{Effects of BaSC06 on Production of Immune Cytokines in lleum Mucosa}

The level of pro-inflammatory cytokines IL- 6 and chemokine MCP1 in the ileum mucosa in the semisubstitute antibiotic group (Anti+Ba group) was significantly reduced by $86.0 \%(P<0.05)$ and $32.3 \%(P$ $<0.05)$ (Fig. 4A), while IL-6 and IL-8 in Ba group were significantly reduced by $42.3 \%(P<0.05)$ and $13.6 \%$ $(P<0.05)$, respectively (Fig. 4A). Also, IL-8 in the Anti+Ba group and MCP1 in the Ba group had no significant changes compared with the control group ( $P>0.05$ ) (Fig. 4A). However, IL-6 in the Ba group was significantly increased compared to the Anti+Ba group $(P<0.05)$ (Fig. 4A). These results showed that BaSC06 instead of antibiotics could alleviate intestinal inflammation by inhibiting the production of proinflammatory cytokines and chemokine in fattening pigs.

\subsubsection{Effects of BaSC06 on Expression of Key Proteins in M1/M2 Macrophages Polarization Signaling Pathways}

The expression of polarized marker protein inducible nitric oxide synthase (iNOS) of M1 macrophages of the two groups supplemented with BaSC06 increased significantly $(P<0.05)$ (Fig. 4B), which indicated that $\mathrm{BaSC} 06$ could promote the polarization of intestinal macrophages to M1 phenotype. What's more, the expression of Arginase (Arg), a marker protein of M2 macrophages, in the Ba group was also significantly up-regulated compared with the Anti group and the Anti + Ba group $(P<0.05)$ (Fig. 4B). There was no significant difference in the expression of $\mathrm{M} 2$ polarized marker protein Mannose receptor (MR) among three groups $(P>0.05)$ (Fig. 4B). The results showed that BaSC06 could promote M1 and M2 polarization of intestinal macrophage simultaneously.

There were no significant changes in the signal transduction and expression of activators of transcription signal transducer and activator of transcription (STAT1) and p-STAT1 and interferon regulator IFNregulatory factor 3 (IRF3) with BaSC06 supplemented in the feed ( $P>0.05)$ (Fig. 4C). The expression of phosphorylated IRF3 protein in Anti+Ba group had an insignificant upward trend $(P=0.061)$. The same result in the expression of STAT6 ( $P>0.05$ ) (Fig. 4D) was observed, whereas the expression of STAT3 
protein in Ba group was significantly increased $(P<0.05)$ (Fig. 4D). This result indicated that the promotion of M1 polarization of macrophages induced by BaSC06 was not involved in the STAT1 and IRF3 signaling pathway, and the increased M2 polarization by activation of STAT3, not by STAT6.

\subsection{Effects of BaSC06 on Intestinal Microbiota}

As shown in Fig. 5A, in addition to the unclassified bacteria, there are five main categories of Verrucomicroba, Spirochaetes, Proteobacteria, Bacteroidetes and Firmicutes in the cecum flora of fattening pigs. In the Class level, we further analysis of the effect of probiotics BaSC06 on Bacteroides and Flavobacteria in Bacteroidetes, (Fig. 5B). Bacteroides account for the vast majority of Bacteroidetes, $71.09 \%, 83.50 \%$ and $67.66 \%$, respectively, while the amount of Flavobactera is very small (Fig. 5B). Fattening pigs' Firmicutes in caecum could be divided into Bacilli, Clostridia and Erysipelotrichia. Bacilli accounted for Firmicutes of $67.98 \%, 82.13 \%$ and $84.48 \%$, respectively in three groups (Fig. 5C). Wherein, Erysipelotrichi occupy $13.9 \% 6.77 \%$ and $6.15 \%$, respectively, in Firmicutes (Fig. 5C). The result shows that BaSC06 could significantly increase the proportion of Gram-positive bacteria (Firmicutes), and reduce the proportion of Gram-negative bacteria (Bacteroides, Proteobacteria) without affecting the diversity of intestinal microbiota of fattening pigs.

\section{Discussion}

The problem of residues and resistance caused by antibiotics abusing has caused widespread concerns. Probiotics, as a green feed additive with the potential to replace antibiotics [14], are widely promoted due to their ability to significantly improve production efficiency and welfare of the animal husbandry industry [15]. Our previous study has found that, instead of aureomycin, BaSC06 increased the growth performance of piglets by improving average daily gain [16]. In the current study, the similar results were obtained, which are consistent with other results. Supplementation of $0.2 \%$ probiotics Bacillus (including Bacillus subtilis, Bacillus coagulans, and Lactobacillus acidophilus) in the diet could significantly increase the average daily gain of fattening pigs, but has no significant effect on the average daily feed intake and F/G [17]. Balasubramanian et al. (2016) found that the Bacillus compound not only significantly increased the average daily weight gain, but also improved F/G [18]. It indicated that Probiotic $\mathrm{BaSC} 06$ can replace antibiotics both in piglets and fattening pigs. Many studies have been verified that the beneficial effects of probiotics on animal growth is related to improve intestinal health, including digestion absorption function, antioxidative capacity, immune function, and microbiota composition [19-22].

The activity of digestive enzymes reflects the digestion and absorption capacity of feed nutrients in monogastric animals. Our research found that BaSC06 could increase trypsin and amylase activity in the intestine of fattening pigs, thereby promoting the digestion and utilization of protein and carbohydrates in the diet. Other study also found that Brevibacillus brevis FJAT-1501-BPA, Bacillus subtilis supplementary significantly improved the feed conversion rate and growth performance of weaning piglets through regulating trypsin, amylase, and cellulase detected in the feces $[23,24]$. Besides, our results indicated that 
the intestinal chymotrypsin and lipase activities in the group supplemented with BaSC06 are lower, which may be related to the consumption of a large amount of lipase and chymotrypsin after feeding BaSC06 to promote the hydrolysis of fat and protein in the body. Similar results were found in broilers which were feed with compound probiotics (Bacillus subtilis, Lactobacillus acidophilus, and Bacillus licheniformis) [25]. In addition, BaSC06 could increase the sucrase and lactase activity without affecting maltase activity, then further improve the absorption of nutrients. Goyal et al. (2013) have found that feeding rats with Lactobacillus La1 can increase sucrase activity in the intestine; what's more, pre-oral administration of LGG increases sucrase and lactase activity and helps mice effectively resist oxidative stress induced by flagellate infection [26]. We speculate that the changes in digestive enzyme activity detected in this study may be due to the secretion of probiotics and the stimulation of endogenous enzyme synthesis in intestines [27].

When the protein is hydrolyzed in the intestine to produce free amino acids, a large number of di- and tripeptides are also produced, which are the main absorption forms of protein [28]. y- Glutamytransferase can regulate the glutamine nutrition metabolism of intestinal epithelial cells, improve the uptake, transport, and utilization of amino acids by cells, and thus maintain intestinal mucosal integrity, improve intestinal health, and create favorable conditions for digestion and absorption $[29,30]$. Transporters are involved in the transmembrane transport of di- and tripeptides. Current studies have found that there are at least five small peptide transporters, of which the most widely studied are PEPT1 and PEPT2. PEPT1 is highly expressed in the brush border of the jejunum epithelium of pigs and mediates intestinal absorption of di- and tripeptides [31, 32]. Studies have shown that when continuous feeding of Bacillus subtilis, the expression level of small peptide transporter PEPT1 mRNA in the ileum mucosa of broiler chickens is significantly higher than that in the basic diet group and the antibiotics group. The digestion and absorption mainly by upregulating the expression of the small peptide transporter PEPT1 in the ileum improves the absorption and transport of small peptides by the intestine [33]. However, we found that mixed BaSC06 and Kitasamycin did not affect the activity of y-Glutamytransferase in intestinal mucosal cells of fattening pigs, but BaSC06 alone decreased the activity of $y$-Glutamytransferase significantly. BaSC06 may enhance the cell's absorption capacity, resulting in reduced demand for $\mathrm{Y}^{-}$ Glutamytransferase. Interestingly, we also found that adding BaSC06 alone could increase the expression of s PEPT1 mRNA by 6 to 7 times, which may promote the transport and absorption of small peptides in vivo. Furthermore, $\mathrm{Na}+/ \mathrm{K}+-\mathrm{ATPase}$ can hydrolyze ATP to help the body obtain energy, transport $\mathrm{Na}+$ against chemical gradients, and regulate the electrochemical balance of $\mathrm{Na}+$ and $\mathrm{K}+$ inside and outside the intestinal cell membrane. It is particularly important to maintain $\mathrm{Na+-dependent} \mathrm{transporters} \mathrm{such} \mathrm{as}$ SGLT1 (Sodium-dependent glucose transporter 1) and GLUT2 (Glucose transporter 2), distributing in the small intestine epithelium of animals. They both mediate the transport of glucose molecules across the brush border of the intestinal epithelium [34,35]. Therefore, AKPase and $\mathrm{Na}+\mathrm{K}+$-ATPase activity and the expression of glucose transporter on intestinal mucosa can reflect the absorption function of small intestinal epithelial cells well. We observed that instead of antibiotic, BaSC06 increased the activity of $\mathrm{Na}+/ \mathrm{K}+-A T P a s e$ in jejuna mucosa and the expression of SGLT1, which indicated that as an alternative of antibiotic, BaSC06 could influence the carbohydrates metabolism and improve the absorption ability of 
intestinal epithelial cells, while the certain further research was needed to clarify the certain effects of BaSC06.

With the generation of radicals during the process of energy metabolism, the intestine, as an organ that connects with the outside, is extremely easy to attack by excessive oxygen free radicals, which can trigger cell membrane lipid peroxidation, protein denaturation, and DNA lysis. Oxidative stress causes damage to intestinal mucosal cells and intestinal tissues of animals and metabolic disorders of the body, and then induces a series of intestinal diseases, leading to abnormal intestinal functions [36-38]. Normally, the levels of oxidation and reduction in the body maintain a dynamic balance, which can be broken by a variety of endogenous and exogenous harmful stimuli. If the levels of free radicals and ROS exceed the reduction level of the antioxidant system, the antioxidant capacity of the body decreases, resulting in oxidative damage to biological macromolecules [39]. A large number of studies have shown that free radicals can cause peroxidative damage to cell membrane lipids and produce MDA which involved in attacking polyunsaturated fatty acids in biological cell membranes, further triggering lipid peroxidation, leading to more severe oxidative stress damage $[40,41]$. However, many studies have shown that probiotics have excellent antioxidant functions. For instance, Zhao et al. (2017) found that Lactobacillus casei significantly reduced MDA content in the serum of pigs and relieved Lipopolysaccharide (LPS)induced oxidative stress [42]. In the present study, BaSC06 can reduce the MDA content in the intestinal mucosa of fattening pigs by increasing CAT and GSH-Px, indicating that the strain has good antioxidant activity and prevent intestinal mucosa from oxidative stress damage. This is consistent with previous BaSC06 results in piglets and rats in our group $[16,43]$.

In addition to antioxidant enzymes, some important phase Il detoxifying enzymes such as NQ01, GST, HO-1, and Y-GCS can also play an antioxidant role in the antioxidant process. NQO1 is a type of flavin protease that is ubiquitous in eukaryotic cells. It can catalyze the reduction of quinone compounds and avoid the formation of unstable semiquinones. Therefore, NQO1 can protect cells from oxidative damage caused by biological heterologous substances such as quinones [44]. HO-1 is an inducible phase II detoxifying enzyme [45], the expression of which is regulated by Nrf2 [46], and its increase in expression can also be used as an adaptive mechanism for cell self-protection under oxidative stress $[47,48]$. Wang et al. (2017) found that HO-1 is a sensitive index for oxidative stress [9]. In this study, the expression of NQ01 mRNA in the BaSC06 group was significantly up-regulated, while the HO-1 gene showed the opposite trend. The up-regulation of NQ01 gene expression indicates that BaSC06 enhances the selfprotection ability of intestinal mucosal cells. The decrease of HO-1 indicates a reduction in oxidative stress, which was resulted from higher antioxidant enzymes activity and NQ01 gene expression induced by $\mathrm{BaSC06}$ treatment. The above results suggest that BaSC06 can regulate the jejuna mucosa phase II detoxifying enzyme-related genes at the transcription level, improve the intestinal antioxidant function, and effectively resist oxidative damage induced by biological heterologous substances and other stress factors.

The Nrf2/Keap1 signaling pathway is important for the body to resist oxidation. In the normal state, the binding of Nrf2 and its inhibitor Keap1 is in resting, while stimulated by oxidative stress factors, Nrf2 and 
Keap1 rapidly dissociate and move into the nucleus, recognize and bind to antioxidant response elements (AREs). Besides, the transcription and translation of cytoprotective genes such as downstream antioxidase and phase II detoxifying enzymes are initiated, thereby improving the cell's antioxidant capacity $[49,50]$. Recent studies have shown that the mechanism by which microorganisms activate the Nrf2 signaling pathway protects the intestinal epithelium from oxidative stress and other endogenous stimuli, and maintains intestinal epithelial cell homeostasis and cell self-protection [51-53]. Chowdhury et al. (2014) found that Bacillus megaterium RB-05 by secreting low fucose to up-regulate the translation and accumulation of Nrf2 and Keap1 in nuclear and then stimulate the expression of downstream transcription factors like HMOX1, NQ01, GSTA2, SOD1 and GPX1 [54]. However, no significant change in the expression of Nrf2 and Keap1 was observed, but we found that BaSC06 significantly up-regulated the expression of phosphorylated Nrf2 protein in the jejuna mucosa of fattening pigs. We also observed similar results on intestinal porcine epithelial cells-1 (IPEC-1) cell experiments [9]. Therefore, we speculate that the activation of the Nrf2/Keap1 signaling pathway indicated by higher p-Nrf2 protein expression in the jejunal mucosa of fattening pigs induced by BaSC06 could promote Nrf2 nuclear translocation, then start transcription and translation of downstream genes (such as the aforementioned antioxidase GSHPx and CAT, phase II detoxifying enzymes, etc.), resulting in improving the total intestinal antioxidant capacity, protecting the intestine from oxidative damage, maintaining the normal physiological function of the intestine, and ultimately promoting digestion and absorption. These results are similar to that of previous studies on antioxidants of other probiotics $[53,55]$.

In addition to increasing antioxidant enzymes, probiotics can also reduce oxidative stress by reducing ROS production. Reactive nicotinamide adenine dinucleotide phosphate oxidase (NOX) is the main source of reactive oxygen species in vitro. NOX is a complex composed of the catalytic subunit gp91 phox and the regulatory subunits $\mathrm{P} 22^{\text {phox }}, \mathrm{P} 47^{\text {phox }}, \mathrm{P} 67^{\text {phox }}, \mathrm{P} 40^{\text {phox }}$, and Rac [56]. Among these subunits, $\mathrm{P} 47^{\text {phox }}$ is considered to play a key role in regulating NOX activity [57]. When stimulated by stress factors such as cytokines, hormones, and inflammatory factors, $\mathrm{P} 47^{\text {phox }}$ undergoes phosphorylation and configuration changes. Subsequently, $\mathrm{P} 47^{\text {phox }}$ binds to subunits such as $\mathrm{P} 67^{\mathrm{phox}}$ in the cytoplasm and is transferred to the cell membrane. The catalytic subunit gp $91^{\text {phox }}$ and the regulatory subunit $\mathrm{P} 22^{\text {phox }}$ bind and interact with each other, eventually activating NOX and generating a large amount of ROS [58-60]. Probiotics alter NOX activity by regulating P47 $7^{\text {phox }}$ expression. Rashid et al. (2014) showed that oral administration of probiotics could protect $\mathrm{P} 47^{\text {phox }}$ protein expression in rats from vascular endothelial dysfunction induced by common bile duct infarction [61], while Tapia-Paniagua et al. (2015) also suggested that probiotic SpPdp11 down-regulating the transcription of NOX in Solea senegalensis [62]. In our former study, we didn't found that BaSC06 replacement affects NOX activity and the expression of $\mathrm{P} 47^{\text {phox }}$ in piglets [16]. However, we found that BaSC06 alone could inhibit the production of excess ROS by down-regulating the expression of NOX active subunit P47 ${ }^{\text {phox }}$ protein in IPEC-1 [9], which is consistent to our present result on fattening pigs.

There are abundant lymphoid tissues in the intestinal mucosa, lymphocytes, plasma cells and macrophages responses to harmful antigens from the gut, such as food toxins, bacteria and viruses. 
Therefore, intestines are the first immune defense system of the digestive system which is known as intestinal mucosa immunity [63]. The interaction between microorganisms and intestinal epithelial cells is the beginning of the host immune response, which can eliminate potential pathogenic microorganisms $[64,65]$. The level of IL-6 and IL-8 in the intestinal mucosa of piglets decreased significantly when Enterococcus faecium was added to the diet [66]. Drouaultholowacz et al. (2006) found that a probiotic with anti-inflammatory effect can effectively prevent intestinal inflammation by increasing the level of anti-inflammatory factors and reducing the level of pro-inflammatory factors [67]. It has been reported that LGG can effectively reduce the level of pro-inflammatory factors in the intestinal mucosa of mice, increase the level of anti-inflammatory factors, and enhance the immune response of mice infected with flagellates [68]. In vitro, it was found that co-culture of Lactobacillus reuteri and IPEC-1 could significantly inhibit the overexpression of TNF-a and IL- 6 caused by Escherichia coli K88 or lipopolysaccharide, and significantly promote the expression of IL-10 [69]. The results in our study also showed that BaSC06 could inhibit the development of intestinal inflammation in fattening pigs by regulating the expression of pro-inflammatory cytokines such as IL-6, IL-8 and MCP1 in the intestinal mucosa. However, other studies have also reported that probiotics themselves can stimulate the immune system of the intestinal mucosa $[70,71]$. For example, Anderson et al. (2016) showed that Lactobacillus fermentum AGR1487 can upregulate the expression of proinflammatory factors by activating the TLR signaling pathway and induce the inflammatory response in sterile rats. The different effects of microorganisms on the intestinal mucosal immune system may be due to the differences between test conditions and strains [72].

As an innate immune cells in the gut, macrophages play a key role in maintaining intestinal homeostasis, regulating cytokine secretion and generating immune response [73-75]. Macrophages can sense the stimulation from microorganisms and activate polarization, while the diversity of microorganisms will lead to the polarization difference of $\mathrm{M} 1$ or $\mathrm{M} 2$ types of macrophages [76-78]. Classical activation of M1 macrophages in response to INF- $\gamma$ is characterized by high ability to express antigens, high expression of iNOS, IL-6, TNF- $\alpha$ and IL-1 $\beta$ mRNA and nitric oxide (NO), which has the ability to kill cells endogenous pathogens and tumor cells [79]. In contrast, activation of M2 macrophages is promoted by various factors (such as IL-4), which is consistent with the high mRNA expression of Arg1, Fizz1, Ym1, and MR, which plays an important role in in the inflammatory response, removing debris, blood vessels generation as well as tissue repairing and reconstruction [80]. In this study, BaSC06 could up-regulate the expression of M1(iNOS) and M2 (Arg) macrophage marker protein simultaneously, which could promote the phagocytosis and sterilization of cells, and enhance the anti-inflammatory and anti-infection ability of cells. Also, Fu et al. (2019) reported that BaSC06 induced M1 phenotype polarization directly and M2 phenotype polarization via modifying microbiota [81]. The polarization imbalance of macrophages, such as the over enhancement of M1 polarization, will lead to immune damage, while the over enhancement of M2 polarization will promote chronic infection, and then induce various diseases [82]. Therefore, we speculated that BaSC06 could maintain the intestinal immune balance of fattening pigs. E. coli Niss/e 1917 can also induce the expression or secretion of pro-inflammatory and anti-inflammatory cytokines in macrophages $[78,83]$. Our previous results of intestinal mucosal cytokines showed that BaSC06 enhanced the immune tolerance of intestinal mucosa in fattening pigs and inhibited the intestinal 
mucosal inflammation. Therefore, the expression of macrophage polarization marker protein may adapt to this change in the microenvironment, avoid the extreme situation of M2 type immune tolerance, maintain the balance of $\mathrm{M} 1$ and $\mathrm{M} 2$ polarization, and then promote pro- and anti-inflammatory balance to maintain intestinal homeostasis. Bacillus amyloliquefaciens enhanced phagocytic bactericidal function by inducing M1 polarization of mouse bone marrow-derived macrophages [84]. Bacillus amyloliquefaciens reduced the intestinal inflammation and diarrhea rate by inhibiting the Mitogenactivated protein kinase (MAPK) inflammation signal pathway in piglets [85]. There were no significant changes in the total protein and phosphorylated protein of proteins related to the M1 polarization signaling pathway detected in this study, such as STAT1 and IRF3, which indicates that BaSC06 affect other pathways such as NF-KB [86], IRF5 $[87,88]$ or JAK to activate macrophage M1 polarization. And STAT6 is the major signaling protein of M2 macrophage polarization signaling pathway mediated by IL-4 [86]. Studies have shown that the inhibition of STAT3 leads macrophages polarizes toward the M1 phenotype [89]. In the current study, the expression of STAT3 protein was significantly up-regulated in BaSC06 supplement groups, which indicates that BaSC06 could promote the polarization of macrophages to the M2 phenotype by activating the STAT3 signaling pathway. In vitro, Shiraishi et al. (2012) have found that glucagon-like peptides-1 can up-regulate the expression of phosphorylated STAT3 protein and activate STAT3 to mediate the polarization of macrophages toward the M2 phenotype [90], which is consistent with our results.

Maintaining intestinal microorganisms balance is essential for intestinal development and homeostasis, which further effectively resist pathogenic bacteria. Intestinal microorganisms are mainly classified into about 50 phyla. In the human intestine, Firmicutes and Bacteroidetes are dominant, while Proteobacteria, Actinobacteria, Clostridia etc. only account for a few [91]. We found that the dominant bacteria in the cecum contents of fattening pigs were Firmicutes and Bacteroidetes. The Firmicutes spherical or rodshaped mainly includes gram-positive bacteria with low $\mathrm{G}+\mathrm{C}$ content. The most important class is Bacillus, including Bacillus, Enterococcus, Lactobacillus, Lactococcus and other beneficial bacteria, which play an important role in maintaining the gut health of animals [92]. Bacteroidetes are Gram-negative bacteria and the second-largest bacterium, live in the intestines of healthy people or animals.

Proteobacteria are also Gram-negative bacteria, which is the largest of the bacteria bacterium, including many pathogenic bacteria, such as E. coli, Salmonella, Vibrio, Helicobacter and so on. The beneficial effects of probiotics may come from inhibiting the growth of pathogenic bacteria and promoting the growth of beneficial flora in the gastrointestinal tract. Probiotic colonization and its effect on members of the intestinal microbiota are highly species-specific. Enterococcus faecium and Lactobacillus can reduce the abundance of $E$. coli and increase the abundance of anaerobic bacteria in piglets' ileum and cecum [93]. Bacillus can occupy the surface of intestinal mucosa together with other anaerobic microorganisms to form a biological barrier to protect the intestinal mucosa and prevent the invasion of pathogenic bacteria. They can also inhibit the growth and reproduction of harmful microorganisms such as Clostridium, improve the disease resistance of piglets, and then improve its growth performance [11,94]. Bacillus increases the abundance of Bacillus, Bifidobacterium, and Lactobacillus in the gut of pigs, while the abundance of $E$. coli is relatively reduced [95-98]. This study found that compared with Anti group, 
BaSC06 did not affect the diversity of the intestinal microorganisms of the fattening pigs, but it could improve the structure of it, especially significantly increase the proportion of Gram-positive bacteria (Firmicutes), and reduce the proportion of gram-negative bacteria (Bacteroidetes, Proteobacteria). Therefore, the probiotic BaSC06 can promote the balance of the intestinal microorganisms, improve the microbial barrier function, strengthen the dominant position of the Firmicutes, and significantly reduce gram-negative pathogen infection, thereby promote the intestinal health of fattening pigs.

\section{Conclusions}

In summary, oral administration of BaSC06 as a substitute for antibiotics could improve growth performance of fattening pigs via increasing intestinal enzymatic activities of digestion and absorption and expression of transporters and antioxidant capacity indicated by the increased antioxidant enzymes activity by activating the Nrf2 signaling pathway and the decreased ROS production, enhancing the intestinal integrity, regulating the intestinal immune function, promoting macrophage polarization of M1 type and M2 type by activating the STAT3 signaling pathway, and improving intestinal microbiota composition. Encouraging the assessment of BaSC06 in further research of intestinal development, injured intestinal repair and stem cell differentiation. Therefore, according to our research, Bacillus amyloliquefaciens $\mathrm{SCO} 6$ could be used as an alternative to antibiotics in piglets and fattening pigs.

\section{List Of Abbreviations}




\begin{tabular}{|c|c|}
\hline BaSC06 & Bacillus amyloliquefaciens SCO6 \\
\hline ADFI & Average Daily Feed Intake \\
\hline ADG & Average Daily Gain \\
\hline AKPase & Alkaline phosphatase \\
\hline Arg & Arginase \\
\hline ASCT & ASC-type animo acid transporter \\
\hline $\mathrm{B}^{0} \mathrm{AT} 1$ & $\mathrm{~B}^{0}$-type amino acid transporter 1 \\
\hline CAT & Catalase \\
\hline CAT & Catalase \\
\hline EAAC1 & Excitatory amino acid carrier 1 \\
\hline$F / G$ & Feed/Gain \\
\hline GAPDH & Glyceraldehyde-3-phosphate dehydrogenase \\
\hline GLUT2 & Glucose transporter 2 \\
\hline GSH & Glutathione \\
\hline GSH-Px & Glutathione peroxidase \\
\hline GST & Glutathione S-transferase \\
\hline HO-1 & Hemeoxygenase-1 \\
\hline HRP & Horseradish peroxidase \\
\hline IL & Interleukin \\
\hline iNOS & Inducible nitric oxide synthase \\
\hline IPEC & Intestinal porcine epithelial cells \\
\hline IRF3 & IFN-regulatory factor 3 \\
\hline Keap1 & Kelch-like epichlorohydrin-associated protein 1 \\
\hline LAT1 & L-type amino acid transporter 1 \\
\hline LPS & Lipopolysaccharide \\
\hline MAPK & Mitogen-activated protein kinase \\
\hline MCP1 & Monocyte Chemotactic Protein 1 \\
\hline MDA & Methane dicarboxylic aldehyde \\
\hline MR & Mannose receptor \\
\hline
\end{tabular}




\begin{tabular}{ll}
$\mathrm{Na}^{+}, \mathrm{K}^{+}$-ATPase & Sodium potassium ATPase \\
\hline NOX & NADPH oxidase \\
\hline NQ01 & NAD (P)H: Quinone oxidoreductase 1 \\
\hline Nrf2 & Nuclear transcription factor-erythroid 2-related factor \\
\hline PEPT1 & Peptide transporter 1 \\
\hline SGLT1 & Sodium-dependent glucose transporters 1 \\
\hline STAT & Signal transducer and activator of transcription \\
\hline T-AOC & Total Antioxidant Capacity \\
\hline TNF-a & Tumor Necrosis Factor-a \\
\hline T-SOD & Total Superoxide Dismutase \\
\hline V/C & The height of the villi/the depth of the crypt \\
\hline Y-GT & Y-glutamyl transpeptidase
\end{tabular}

\section{Declarations}

\section{Ethics approval and consent to participate:}

All the experimental procedures were approved by and performed according to the guidelines of the Animal Care and Use Committee of Zhejiang University.

\section{Consent for publication:}

Not applicable

\section{Availability of data and materials:}

The datasets used and/or analysed during the current study are available from the corresponding author on reasonable request.

\section{Competing interests $\llbracket$}

The authors declare that they have no competing interests.

\section{Funding:}

This study is supported by the National Natural Science Foundation of China (No. 31472128 and

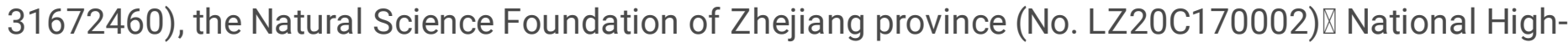
Tech R\&D Program (863) of China (No. 2013AA102803D), the Major Science and Technology Project of Zhejiang Province (No. 2006C12086), PRC. 


\section{Authors' contributions:}

$\mathrm{XC}$ conceived and designed the experiments; $\mathrm{XC}, \mathrm{LT}$ and $\mathrm{ZZ}$ conducted the experiments; $\mathrm{XC}, \mathrm{LT}$ and $\mathrm{BW}$ analyzed the data and made the figures; LT wrote the manuscript; $Y Z, Q W$ and PZ revised the manuscript. All the authors reviewed and approved the final version of the manuscript and agreed to be accountable for the content of the work.

\section{Acknowledgements:}

Not applicable.

\section{References}

1. Kim JW, Koo B, Nyachoti CM: Net energy content of canola meal fed to growing pigs and effect of experimental methodology on energy values. J Anim Sci 2018, 96(4):1441-1452.

2. Michiels J, Possemiers, S. , Degroote, J. , Ovyn, A. , De, S. S. , \& Nakamura, N.: Feeding Bacillus subtilis C-3102 to sows and suckling piglets and to weaned piglets improves parameters of gut health and feed:gain ratio in weaners. Journal of Animal science 20162016,94.(3):135-137. Olhttps://doi.org/10.2527/jas.2015-9763

3. Piskoríková MJJoAAN: The efficacy of the probiotic feed additive Calsporin? (Bacillus subtilis C3102) in weaned piglets: combined analysis of four different studies. Journal of Applied Animal Nutrition 2012, 1:e2. https://doi.org/10.1107/S0907444902001762

4. Yi H, Wang L, Xiong Y, Wen X, Wang Z, Yang X, Gao K, Jiang ZJJoas: Effects of Lactobacillus reuteri LR1 on the growth performance, intestinal morphology, and intestinal barrier function in weaned pigs. 2018, 96(6):2342-2351. https://doi.org/10.1093/jas/sky129

5. A R Gould BKM, W H Elliott: Release of extracellular enzymes from Bacillus amyloliquefaciens. Journal of Bacteriology 1975, 122(1):34-40. https://doi.org/10.1128/JB.122.1.34-40.1975

6. Gracia MI, Araníbar, M.J., Lázaro, \& R.: a-Amylase Supplementation of Broiler Diets Based on Corn. Poultry Science 82:436-442 2003. https://doi.org/10.1093/ps/82.3.436

7. Lee YJ, Kim BK, Lee BH, Jo KI, Lee NK, Chung CH, Lee YC, Lee JW: Purification and characterization of cellulase produced by Bacillus amyoliquefaciens DL-3 utilizing rice hull. $J$ Bioresource Technology 2008, 99(2):378-386. https://doi.org/ 10.1134/S0006297907040141

8. Hong HA, Hong DL, Cutting SM: The use of bacterial spore formers as probiotics. J Fems Microbiology Reviews 2005(4):4. https://doi.org/ 10.1016/j.femsre.2004.12.001

9. Wang Y, Wu Y, Wang Y, Fu A, Gong L, Li W, Li YJAm, biotechnology: Bacillus amyloliquefaciens SC06 alleviates the oxidative stress of IPEC-1 via modulating Nrf2/Keap1 signaling pathway and decreasing ROS production. 2017, 101(7):3015-3026. https://doi.org/10.1007/s00253-016-8032-4

10. Hu S, Cao X, Wu Y, Mei X, Xu H, Wang Y, Zhang X, Gong L, Li WJFim: Effects of probiotic Bacillus as an alternative of antibiotics on digestive enzymes activity and intestinal integrity of piglets. 2018, 9:2427. https://doi.org/10.3389/fmicb.2018.02427 
11. Du W, Xu H, Mei X, Cao X, Gong L, Wu Y, Li Y, Yu D, Liu S, Wang YJBm: Probiotic Bacillus enhance the intestinal epithelial cell barrier and immune function of piglets. 2018, 9(5):743-754. https://doi.org/10.3920/BM2017.0142

12. Qin C, Gong L, Zhang X, Wang Y, Wang Y, Wang B, Li Y, Li WJAn: Effect of Saccharomyces boulardii and Bacillus subtilis B10 on gut microbiota modulation in broilers. 2018, 4(4):358-366. https://doi.org/10.1016/j.aninu.2018.03.004

13. Zhang X, Shu M, Wang Y, Fu L, Li W, Deng B, Liang Q, Shen WJWJoM, Biotechnology: Effect of photosynthetic bacteria on water quality and microbiota in grass carp culture. 2014, 30(9):25232531. https://doi.org/10.1007/s11274-014-1677-1

14. Mingmongkolchai S, Panbangred WJJoam: Bacillus probiotics: an alternative to antibiotics for livestock production. 2018, 124(6):1334-1346. https://doi.org/ 10.1111/jam.13690

15. Chen L, Zhou C, Liu G, Jiang H, Lu Q, Tan Z, Wu X, Fang JJJoFA, Environment: Application of lactic acid bacteria, yeast and bacillus as feed additive in dairy cattle. 2013, 11:626-629. https://doi.org/10.1146/annurev-food-022811-101247

16. Wang Y, Wu Y, Wang B, Cao X, Fu A, Li Y, Li WJAE: Effects of probiotic Bacillus as a substitute for antibiotics on antioxidant capacity and intestinal autophagy of piglets. 2017, 7(1):52. https://doi.org/10.1186/s13568-017-0353-x

17. Chen Y, Min B, Cho J, Kwon O, Son K, Kim H, Kim IJA-ajoas: Effects of dietary Bacillus-based probiotic on growth performance, nutrients digestibility, blood characteristics and fecal noxious gas content in finishing pigs. 2006, 19(4):587-592. https://doi.org/10.5713/ajas.2006.587

18. Balasubramanian B, Li T, Kim IHJRBdZ: Effects of supplementing growing-finishing pig diets with Bacillus spp. probiotic on growth performance and meat-carcass grade qualitytraits. 2016, 45(3):93100. https://doi.org/ 10.1590/S1806-92902016000300002

19. Wang J, Ni X, Wen B, Zhou Y, Liu L, Zeng Y, Zhao W, Khalique A, Wang P, Pan KJAM et al: Bacillus strains improve growth performance via enhancing digestive function and anti-disease ability in young and weaning rex rabbits. 2020:1-12. https://doi.org/10.1007/s00253-020-10536-9

20. Ward TL, Weber BP, Mendoza KM, Danzeisen JL, Llop K, Lang K, Clayton JB, Grace E, Brannon J, Radovic IJm: Antibiotics and host-tailored probiotics similarly modulate effects on the developing avian microbiome, mycobiome, and host gene expression. 2019, 10(5):e02171-02119. https://doi.org/ 10.1128/mBio.02171-19

21. Hu Y, Dun Y, Li S, Zhao S, Peng N, Liang YJA-Ajoas: Effects of Bacillus subtilis KN-42 on growth performance, diarrhea and faecal bacterial flora of weaned piglets. 2014, 27(8):1131. https://doi.org/10.5713/ajas.2013.13737

22. Sokale A, Menconi A, Mathis G, Lumpkins B, Sims M, Whelan R, Doranalli KJPs: Effect of Bacillus subtilis DSM 32315 on the intestinal structural integrity and growth performance of broiler chickens under necrotic enteritis challenge. 2019, 98(11):5392-5400. https://doi.org/10.3382/ps/pez368

23. Che J, Ye S, Liu B, Deng Y, Chen Q, Ge C, Liu G, Wang JJAvL: Effects of Brevibacillus brevis FJAT1501-BPA on growth performance, faecal microflora, faecal enzyme activities and blood parameters 
of weaned piglets. 2016, 109(12):1545-1553. https://doi.org/ 10.1007/s10482-016-0756-8

24. Liu J, Cao S, Liu J, Xie Y, Zhang HJA-Ajoas: Effect of probiotics and xylo-oligosaccharide supplementation on nutrient digestibility, intestinal health and noxious gas emission in weanling pigs. 2018, 31(10):1660. https://doi.org/ 10.5713/ajas.17.0908

25. Zhi-gang T, Naeem M, Chao W, Tian W, Yan-min ZJJoA, Science P: Effect of dietary probiotics supplementation with different nutrient density on growth performance, nutrient retention and digestive enzyme activities in broilers. 2014, 24:1309-1315. http://www.thejaps.org.pk/docs/v-245/06.pdf

26. Goyal N, Rishi P, Shukla GJWJoM, Biotechnology: Lactobacillus rhamnosus GG antagonizes Giardia intestinalis induced oxidative stress and intestinal disaccharidases: an experimental study. 2013, 29(6):1049-1057. https://doi.org/ 10.1007/s11274-013-1268-6

27. Mohapatra S, Chakraborty T, Prusty A, Das P, Paniprasad K, Mohanta KJAN: Use of different microbial probiotics in the diet of rohu, Labeo rohita fingerlings: effects on growth, nutrient digestibility and retention, digestive enzyme activities and intestinal microflora. 2012, 18(1):1-11. https://doi.org/ 10.1111/j.1365-2095.2011.00866.x

28. Wu GJF, function: Dietary protein intake and human health. 2016, 7(3):1251-1265. https://doi.org/ $10.1039 / \mathrm{c} 5 f \circ 01530 \mathrm{~h}$

29. Borud O, Mortensen B, Mikkelsen IM, Leroy P, Wellman M, Huseby NEJljoc: Regulation of $Y$ glutamyltransferase in cisplatin-resistant and-sensitive colon carcinoma cells after acute cisplatin and oxidative stress exposures. 2000, 88(3):464-468. https://doi.org/10.1002/10970215(20001101)88:33.0.CO;2-F

30. Viña J, Puertes I, Estrela J, Viña J, Galbis JJBJ: Involvement of Y-glutamyltransferase in amino-acid uptake by the lactating mammary gland of the rat. 1981, 194(1):99-102. https://doi.org/10.1042/bj1940099

31. Winckler C, Breves G, Boll M, Daniel HJJoCPB: Characteristics of dipeptide transport in pig jejunum in vitro. 1999, 169(7):495-500. https://doi.org/ 10.1007/s003600050247

32. Daniel HJARP: Molecular and integrative physiology of intestinal peptide transport. 2004, 66:361384. https://doi.org/ 10.1146/annurev.physiol.66.032102.144149

33. Alarcon MFFJP, Genome A: Replacement of Antibiotic Growth Promoter by Probiotic in the Diet Increases Intestinal Peptide Transporter RNA Expression in Broilers. 2014. https://pag.confex.com/pag/xxii/webprogram/Paper13306.html

34. Ait-Omar A, Monteiro-Sepulveda M, Poitou C, Gall ML, Cotillard A, Gilet J, Garbin K, Houllier A, Chateau D, Lacombe AJD: GLUT2 Accumulation in Enterocyte Apical and Intracellular Membranes A Study in Morbidly Obese Human Subjects and ob/ob and High Fat-Fed Mice. 60(10):p.2598-2607. https://doi.org/ 10.2337/db10-1740

35. Cheeseman CIJJoP: The facilitated component of intestinal glucose absorption. 2002, 539:5S-5S. https://doi.org/ 10.1111/j.1469-7793.2001.0585h.x 
36. Harman DJM, Biochemistry C: Free radicals in aging. 1988, 84(2):155-161. https://doi.org/10.1007/BF00421050

37. Secchi A OM, Gebhard M: Effect of endotoxemia on hepatic portal and sinusoidal blood flow in rats. Critical Care 2000, 3(1): 1-1. https://doi.org/ 10.1186/cc552

38. Mitsuoka H S-SGW: MECHANISMS FOR BLOCKADE OF IN VIVO ACTIVATOR PRODUCTION IN THE ISCHEMIC INTESTINE AND MULTI-ORGAN FAILURE. Shock 2000, 14(5):522-527. https://doi.org/10.1097/00024382-200014050-00005

39. Laval JJPB: Role of DNA repair enzymes in the cellular resistance to oxidative stress. 1996, 44(1):1424. https://doi.org/10.1007/s002920050140

40. Shi G F ALJ, Jiang B , et al. : Alpinia protocatechuic acid protects against oxidative damage in vitro and reduces oxidative stress in vivo. Neuroscience Letters 2006, 403(3):206-210. https://doi.org/ 10.1016/j.neulet.2006.02.057

41. Gebretsadik G SD, Yimer G, et al.: The Non-Enzymatic Antioxidant and Level of Oxidative Stress of Tuberculosis Patients in Selected Treatment Center in Addis Ababa Ethiopia. Journal of Tuberculosis Research 2015, 03(3):239-253. https://doi.org/10.4236/jtr.2015.33010

42. Zhao D WT, Yi D, Wang L, Li P, Zhang J, Hou Y, Wu G: Dietary Supplementation with Lactobacillus casei Alleviates Lipopolysaccharide-Induced Liver Injury in a Porcine Model. Mol Sci 2017, 18(12):2535-. https://doi.org/10.3390/ijms18122535

43. Wu Y, Wang B, Xu H, Tang L, Li Y, Gong L, Wang Y, Li WJFim: Probiotic Bacillus attenuates oxidative stress-induced intestinal injury via p38-mediated autophagy. 2019, 10:2185. https://doi.org/ $10.3389 /$ fmicb. 2019.02185

44. Lim JH, Kim K-M, Kim SW, Hwang O, Choi HJJPR: Bromocriptine activates NQ01 via Nrf2-PI3K/Akt signaling: novel cytoprotective mechanism against oxidative damage. 2008, 57(5):325-331. https://doi.org/ 10.1016/j.phrs.2008.03.004

45. Ryter SW, Choi AMJA, Signaling R: Heme oxygenase-1: molecular mechanisms of gene expression in oxygen-related stress. 2002, 4(4):625-632. https://doi.org/ 10.1557/JMR.2004.0382

46. Alam J, Stewart D, Touchard C, Boinapally S, Choi AM, Cook JLJJoBC: Nrf2, a Cap'n'Collar transcription factor, regulates induction of the heme oxygenase-1 gene. 1999, 274(37):26071-26078. https://doi.org/ 10.1074/jbc.274.37.26071

47. Otterbein LE, Soares MP, Yamashita K, Bach FHJTii: Heme oxygenase-1: unleashing the protective properties of heme. 2003, 24(8):449-455. https://doi.org/ 10.1016/S1471-4906(03)00181-9

48. Choi A, Alam JJAjorc, biology m: Heme oxygenase-1: function, regulation, and implication of a novel stress-inducible protein in oxidant-induced lung injury. 1996, 15(1):9-19. https://doi.org/ 10.1165/ajrcmb.15.1.8679227

49. Kwak M-K, Wakabayashi N, Kensler TWJMRF, Mutagenesis MMo: Chemoprevention through the Keap1-Nrf2 signaling pathway by phase 2 enzyme inducers. 2004, 555(1-2):133-148. https://doi.org/ 10.1016/j.mrfmmm.2004.06.041 
50. Itoh K, Chiba T, Takahashi S, Ishii T, Igarashi K, Katoh Y, Oyake T, Hayashi N, Satoh K, Hatayama IJB et al: An Nrf2/small Maf heterodimer mediates the induction of phase II detoxifying enzyme genes through antioxidant response elements. 1997, 236(2):313-322. https://doi.org/ 10.1006/bbrc. 1997.6943

51. Mercante J, Jones RM, Luo L, Moberg K, Neish AS: Intestinal microbiota modulate ROS-dependent epithelial cytoprotection through Nrf2. In.: Federation of American Societies for Experimental Biology; 2011. https://www.fasebj.org/doi/abs/10.1096/fasebj.25.1_supplement.613.2

52. St Clair Ardita C, Jones RM, Mercante JW, Luo L, Gates C, Moberg KH, Neish AS: Commensal Lactobacillus modulate ROS-dependent cytoprotective gene expression in intestinal epithelia. In.: Federation of American Societies for Experimental Biology; 2013. https://www.fasebj.org/doi/abs/10.1096/fasebj.27.1_supplement.131.11

53. Jones RM, Desai C, Darby TM, Luo L, Wolfarth AA, Scharer CD, Ardita CS, Reedy AR, Keebaugh ES, Neish ASJCr: Lactobacilli modulate epithelial cytoprotection through the Nrf2 pathway. 2015, 12(8):1217-1225. https://doi.org/ 10.1016/j.celrep.2015.07.042

54. Chowdhury SR, Sengupta S, Biswas S, Sinha TK, Sen R, Basak RK, Adhikari B, Bhattacharyya AJPO: Bacterial fucose-rich polysaccharide stabilizes MAPK-mediated Nrf2/Keap1 signaling by directly scavenging reactive oxygen species during hydrogen peroxide-induced apoptosis of human lung fibroblast cells. 2014, 9(11). https://doi.org/ 10.1371/journal.pone.0113663

55. Gao D, Gao Z, Zhu GJF, function: Antioxidant effects of Lactobacillus plantarum via activation of transcription factor Nrf2. 2013, 4(6):982-989. https://doi.org/ 10.1039/C3F030316K

56. Lambeth JDJNRI: NOX enzymes and the biology of reactive oxygen. 2004, 4(3):181-189. https://doi.org/ 10.1038/nri1312

57. Zhang L, Dong W, Li Q, Kang L, Zhang L, Lu Y, Zhai XJTJoM-F, Medicine N: Mechanism of p47phoxinduced increase of reactive oxygen species in peripheral blood mononuclear cells from premature infants on oxygen therapy. 2016, 29(21):3490-3494. https://doi.org/ 10.3109/14767058.2015.1135119

58. Park J-BJE, medicine m: Phagocytosis induces superoxide formation and apoptosis in macrophages. 2003, 35(5):325-335. https://doi.org/ 10.1038/emm.2003.44

59. Meng T, Yu J, Lei Z, Wu J, Wang S, Bo Q, Zhang X, Ma Z, Yu JJC, Immunology D: Propofol reduces lipopolysaccharide-induced, NADPH oxidase (NOX 2) mediated TNF-a and IL-6 production in macrophages. 2013. https://doi.org/ 10.1155/2013/325481

60. Casbon AJ, Long ME, Dunn KW, Allen LAH, Dinauer MCJJolb: Effects of IFN-Y on intracellular trafficking and activity of macrophage NADPH oxidase flavocytochrome b558. 2012, 92(4):869-882. https://doi.org/ 10.1189/jlb.0512244

61. Rashid SK, Khodja NI, Auger C, Alhosin M, Boehm N, Oswald-Mammosser M, Schini-Kerth VBJPO: Probiotics (VSL\# 3) prevent endothelial dysfunction in rats with portal hypertension: role of the angiotensin system. 2014, 9(5). https://doi.org/ 10.1371/journal.pone.0097458 
62. Tapia-Paniagua S, Vidal S, Lobo C, de la Banda IG, Esteban M, Balebona M, Moriñigo MJF, immunology s: Dietary administration of the probiotic SpPdp11: effects on the intestinal microbiota and immune-related gene expression of farmed Solea senegalensis treated with oxytetracycline. 2015, 46(2):449-458. https://doi.org/ 10.1016/j.fsi.2015.07.007

63. McGhee J, Kiyono H, Michalek S, Mestecky JJAVL: Enteric immunization reveals a T cell network for IgA responses and suggests that humans possess a common mucosal immune system. 1987, 53(6):537-543. https://doi.org/ 10.1007/BF00415514

64. Lu L, Walker WAJTAjocn: Pathologic and physiologic interactions of bacteria with the gastrointestinal epithelium. 2001, 73(6):1124S-1130S. https://doi.org/ 10.1093/ajcn/73.6.1124S

65. Mackie RI, Sghir A, Gaskins HRJTAjocn: Developmental microbial ecology of the neonatal gastrointestinal tract. 1999, 69(5):1035s-1045s. https://doi.org/ 10.1093/ajcn/69.5.1035s

66. Huang Y, Li Y, Huang Q, Cui Z, Yu D, Rajput IR, Hu C, Li WJPVJ: Effect of orally administered Enterococcus faecium EF1 on intestinal cytokines and chemokines production of suckling piglets. 2012, 32:81-84. https://doi.org/ 10.1603/ME11173

67. Drouault-Holowacz S, Foligné B, Dennin V, Goudercourt D, Terpend K, Burckel A, Pot BJCN: Antiinflammatory potential of the probiotic dietary supplement Lactibiane Tolerance: in vitro and in vivo considerations. 2006, 25(6):994-1003. https://doi.org/ 10.1016/j.clnu.2006.03.006

68. Goyal N, Shukla GJDd, sciences: Probiotic Lactobacillus rhamnosus GG modulates the mucosal immune response in Giardia intestinalis-infected BALB/c mice. 2013, 58(5):1218-1225. https://doi.org/ 10.1007/s10620-012-2503-y

69. Wang Z, Wang L, Chen Z, Ma X, Yang X, Zhang J, Jiang ZJJMB: In Vitro evaluation of swine-derived Lactobacillus reuteri: probiotic properties and effects on intestinal porcine epithelial cells challenged with enterotoxigenic Escherichia coli K88. 2016, 26(6):1018-1025. https://doi.org/ 10.4014/jmb.1510.10089

70. Galdeano CM, Perdigon GJCVI: The probiotic bacterium Lactobacillus casei induces activation of the gut mucosal immune system through innate immunity. 2006, 13(2):219-226. https://doi.org/ 10.1128/CVI.13.2.219-226.2006

71. Bai S, Wu A, Ding X, Lei Y, Bai J, Zhang K, Chio JJPs: Effects of probiotic-supplemented diets on growth performance and intestinal immune characteristics of broiler chickens. 2013, 92(3):663-670. https://doi.org/ 10.3382/ps.2012-02813

72. Anderson RC, Ulluwishewa D, Young W, Ryan LJ, Henderson G, Meijerink M, Maier E, Wells JM, Roy NCJSr: Human oral isolate Lactobacillus fermentum AGR1487 induces a pro-inflammatory response in germ-free rat colons. 2016, 6:20318. https://doi.org/ 10.1038/srep20318

73. Bain CC, Mowat AMJIr: Macrophages in intestinal homeostasis and inflammation. 2014, 260(1):102117. https://doi.org/ 10.1111/imr.12192

74. Mowat AM, Bain CCJJoii: Mucosal macrophages in intestinal homeostasis and inflammation. 2011, 3(6):550-564. https://doi.org/ 10.1159/000329099 
75. Haldar M, Murphy KMJIr: Origin, development, and homeostasis of tissue-resident macrophages. 2014, 262(1):25-35. https://doi.org/ 10.1111/imr.12215

76. Ivanov II, Honda KJCh, microbe: Intestinal commensal microbes as immune modulators. 2012, 12(4):496-508. https://doi.org/ 10.1016/j.chom.2012.09.009

77. Yang Y, Wang X, Huycke T, Moore DR, Lightfoot SA, Huycke MMJTo: Colon macrophages polarized by commensal bacteria cause colitis and cancer through the bystander effect. 2013, 6(5):596. https://doi.org/ 10.1593/tlo.13412

78. Christoffersen TE, Hult LTO, Kuczkowska K, Moe KM, Skeie S, Lea T, Kleiveland CRJP, proteins a: In vitro comparison of the effects of probiotic, commensal and pathogenic strains on macrophage polarization. 2014, 6(1):1-10. https://doi.org/ 10.1007/s12602-013-9152-0

79. Benoit M, Desnues B, Mege J-LJTJol: Macrophage polarization in bacterial infections. 2008, 181(6):3733-3739. https://doi.org/ 10.4049/jimmunol.181.6.3733

80. Satoh T, Takeuchi O, Vandenbon A, Yasuda K, Tanaka Y, Kumagai Y, Miyake T, Matsushita K, Okazaki T, Saitoh TJNi: The Jmjd3-Irf4 axis regulates M2 macrophage polarization and host responses against helminth infection. 2010, 11(10):936. https://doi.org/ 10.1038/ni.1920

81. Fu A, Mo Q, Wu Y, Wang B, Liu R, Tang L, Zeng Z, Zhang X, Li WJF, function: Protective effect of Bacillus amyloliquefaciens against Salmonella via polarizing macrophages to $M 1$ phenotype directly and to M2 depended on microbiota. 2019, 10(12):7653-7666. https://doi.org/ 10.1039/C9F001651A

82. Moore KJ, Sheedy FJ, Fisher EAJNRI: Macrophages in atherosclerosis: a dynamic balance. 2013, 13(10):709-721. https://doi.org/ 10.1038/nri3520

83. Cross ML, Ganner A, Teilab D, Fray LMJFI, Microbiology M: Patterns of cytokine induction by grampositive and gram-negative probiotic bacteria. 2004, 42(2):173-180. https://doi.org/ 10.1016/j.femsim.2004.04.001

84. Ji J, Hu S-L, Cui Z-W, Li W-FJAom: Probiotic Bacillus amyloliquefaciens mediate M1 macrophage polarization in mouse bone marrow-derived macrophages. 2013, 195(5):349-356. https://doi.org/ 10.1007/s00203-013-0877-7

85. Ji J, Hu S, Zheng M, Du W, Shang Q, Li WJLs: Bacillus amyloliquefaciens SC06 inhibits ETECinduced pro-inflammatory responses by suppression of MAPK signaling pathways in IPEC-1 cells and diarrhea in weaned piglets. 2013, 158(1-3):206-214. https://doi.org/ 10.1016/j.livsci.2013.09.017

86. Wang $\mathrm{N}$, Liang H, Zen KJFii: Molecular mechanisms that influence the macrophage M1-M2 polarization balance. 2014, 5:614. https://doi.org/ 10.3389/fimmu.2014.00614

87. Juhas U, Ryba-Stanisławowska M, Szargiej P, Myśliwska JJAiH, Doswiadczalnej EMPHiM: Different pathways of macrophage activation and polarization. 2015, 69. https://doi.org/ 10.5604/17322693.1150133

88. Martinez FOJEjoi: Regulators of macrophage activation. 2011, 41(6):1531-1534. https://doi.org/ 10.1002/eji.201141670 
89. Nakamura R, Sene A, Santeford A, Gdoura A, Kubota S, Zapata N, Apte RSJNc: IL10-driven STAT3 signalling in senescent macrophages promotes pathological eye angiogenesis. 2015, 6:7847. https://doi.org/ 10.1038/ncomms8847

90. Shiraishi D, Fujiwara Y, Komohara Y, Mizuta H, Takeya MJB, communications br: Glucagon-like peptide-1 (GLP-1) induces M2 polarization of human macrophages via STAT3 activation. 2012, 425(2):304-308. https://doi.org/ 10.1016/j.bbrc.2012.07.086

91. Eckburg PB, Bik EM, Bernstein CN, Purdom E, Dethlefsen L, Sargent M, Gill SR, Nelson KE, Relman DAJs: Diversity of the human intestinal microbial flora. 2005, 308(5728):1635-1638. https://doi.org/ 10.1126/science.1110591

92. Haakensen M, Dobson C, Deneer H, Ziola BJljofm: Real-time PCR detection of bacteria belonging to the Firmicutes Phylum. 2008, 125(3):236-241.

93. Hu S, Wang L, Jiang ZJP, letters p: Dietary additive probiotics modulation of the intestinal microbiota. 2017, 24(5):382-387. https://doi.org/ 10.1016/j.ijfoodmicro.2008.04.002

94. SKERMAN VBD, McGowan V, Sneath PHAJIJoS, Microbiology E: Approved lists of bacterial names. 1980, 30(1):225-420. https://doi.org/ 10.1099/00207713-30-1-225

95. Tang W, Qian Y, Yu B, Zhang T, Gao J, He J, Huang Z, Zheng P, Mao X, Luo JJJoas: Effects of Bacillus subtilis DSM32315 supplementation and dietary crude protein level on performance, gut barrier function and microbiota profile in weaned piglets. 2019, 97(5):2125-2138. https://doi.org/ 10.1093/jas/skz090

96. Choi J, Shinde P, Ingale S, Kim J, Kim Y, Kim K, Kwon I, Chae BJLS: Evaluation of multi-microbe probiotics prepared by submerged liquid or solid substrate fermentation and antibiotics in weaning pigs. 2011, 138(1-3):144-151. https://doi.org/10.1016/j.livsci.2010.12.015

97. Wang J, Sun B, Cao Y, Wang CJCp: In vitro fermentation of xylooligosaccharides from wheat bran insoluble dietary fiber by Bifidobacteria. 2010, 82(2):419-423. https://doi.org/ 10.1016/j.carbpol.2010.04.082

98. Luise D, Bertocchi M, Motta V, Salvarani C, Bosi P, Luppi A, Fanelli F, Mazzoni M, Archetti I, Maiorano GJJoas et al: Bacillus sp. probiotic supplementation diminish the Escherichia coli F4ac infection in susceptible weaned pigs by influencing the intestinal immune response, intestinal microbiota and blood metabolomics. 2019, 10(1):74. https://doi.org/ 10.1186/s40104-019-0380-3

\section{Supplementary Table}

Table. S1 List of real-time PCR primers 


\begin{tabular}{|c|c|c|c|}
\hline Gene & Acc. No & Sequence of primers & Length \\
\hline \multirow[t]{2}{*}{ GAPDH } & NM_001206359.1 & FロGGTCGGAGTGAACGGATTT & 245 \\
\hline & & RワATTTGATGTTGGCGGGAT & \\
\hline \multirow[t]{2}{*}{ SGLT1 } & NM_001164021.1 & FロGTGATTTATACGGATACCT & 171 \\
\hline & & RワATAACATTCTTTCTTGATGG & \\
\hline \multirow[t]{2}{*}{ GLUT2 } & NM_001097417.1 & FロAGGCATATCAGGACTCTACT & 77 \\
\hline & & RワACTTGGTTGGAGCAATCT & \\
\hline \multirow[t]{2}{*}{ PEPT1 } & NM_214347.1 & FロCAACATCATCGTGCTTATC & 171 \\
\hline & & R』TCGTCCATATCAAACTGAG & \\
\hline \multirow[t]{2}{*}{$\mathrm{B}^{0} \mathrm{AT} 1$} & DQ231579.1 & FロCTGTCCACAACAACTGCGAGAA & 329 \\
\hline & & $\mathrm{R} \rrbracket$ GAAGACGATGAAAGCCAACCC & \\
\hline \multirow[t]{2}{*}{ ASCT2 } & DQ231578.1 & FロTGGTCTCCTGGATCATGTGGT & 203 \\
\hline & & R』GAAGCGGTAGGGGTTTTTGC & \\
\hline \multirow[t]{2}{*}{ EAAC1 } & NM_001164649.1 & FロATCGTTCAAATCATTATGTG & 167 \\
\hline & & R』TATATCAGTGGCAGAATAAC & \\
\hline \multirow[t]{2}{*}{ LAT1 } & EU390782.2 & FロGGAACTGGGCACCACCATTA & 161 \\
\hline & & R』GCACCACGTAGTTGGCAAAG & \\
\hline \multirow[t]{2}{*}{ GST } & $\mathrm{AB} 000884.1$ & FロCAACCCAGAAGACTGCTCAAG & 159 \\
\hline & & R』GGACCACTCAAGGAATACAGAAG & \\
\hline \multirow[t]{2}{*}{ HO-1 } & NM_001004027.1 & FロAGCTGTTTCTGAGCCTCCAA & 130 \\
\hline & & R』CAAGACGGAAACACGAGACA & \\
\hline \multirow[t]{2}{*}{ NQO1 } & NM_001159613.1 & FロCCAGCAGCCCGGCCAATCTG & 160 \\
\hline & & R』AGGTCCGACACGGCGACCTC & \\
\hline
\end{tabular}

\section{Figures}

Kitasamycin

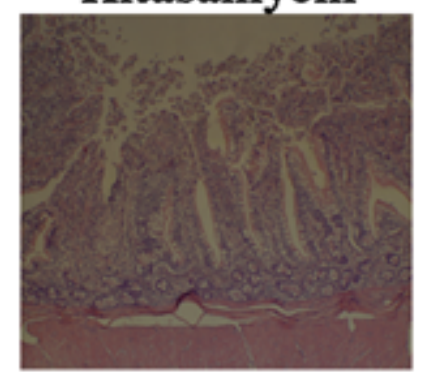

Kitasamycin $+\mathrm{Ba}$

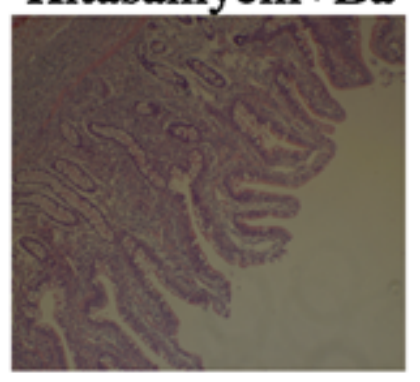

$\mathrm{Ba}$

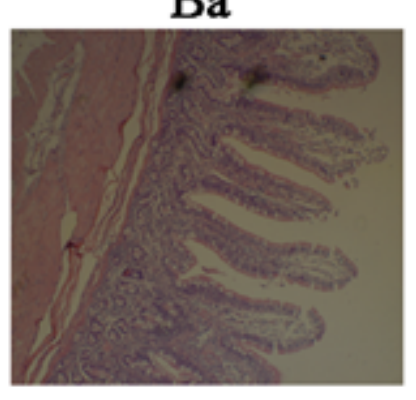

Figure 1 
\Table3 Histomorphometric analysis of ileum mucosa in fattening pigs. (A)H\&E staining, $10 \times 10$ magnification. The values of villus length, crypt length and villus/crypt were calculated and shown in Table 3. Data were shown as mean $\pm S D$ and analyzed using one-way ANOVA Tukey test. Different letters $(a, b, c)$ in each parameter represent significant $(p<0.05)$.

A

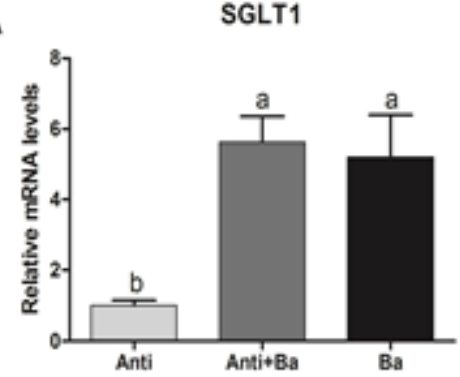

B

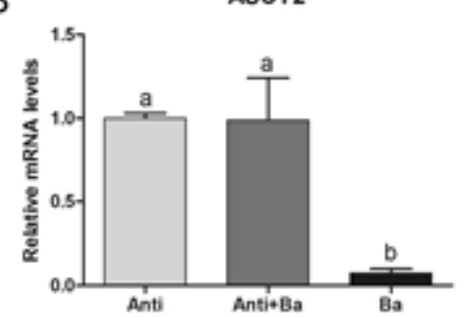

EAAC1
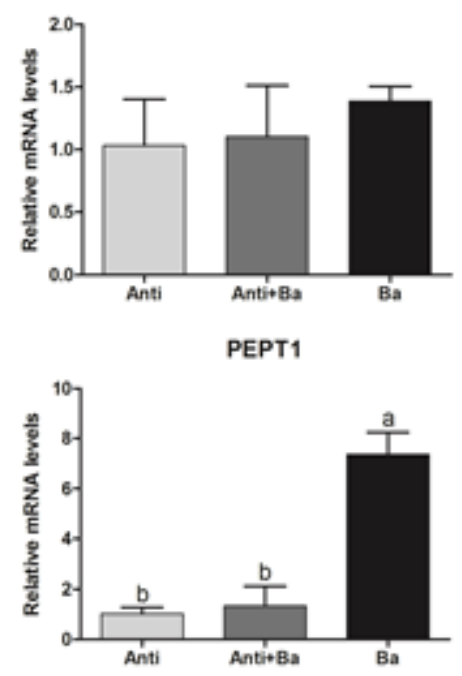

C

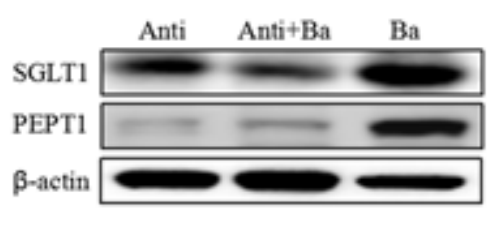

GLUT2

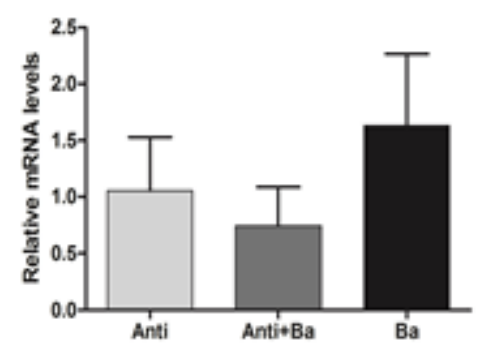

$B^{0}$ AT1

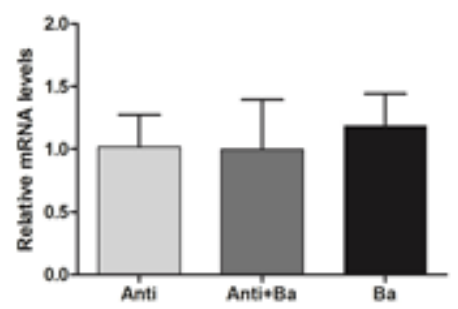

LAT1
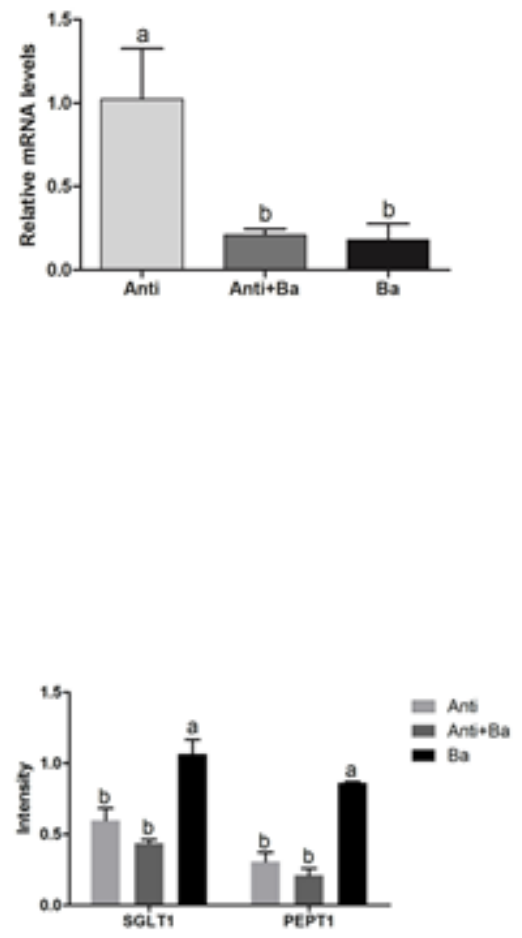

Figure 2 
BaSC06 could increase the expression of glucose transporters and small peptide transporters in jejunum mucosa of fattening pigs. (A囚B)The mRNA levels expression of SGLT1, GLUT1, ASCT2, B0AT1, EAAC1, LAT1 and PEPT1 in jejunum of fattening pigs were analysis with RT-qPCR, the $2-\Delta \Delta C$ t method was used to estimate mRNA abundance. Different letters $(a, b, c)$ in each parameter represent significant $(p<0.05)$. (C) Protein lysates from jejunal mucosa were examined by western blot for SGLT1 and PEPT1 levels. The ratio of SGLT1 and PEPT1 to $\beta$-actin were analyzed using ImageJ. Different letters $(a, b, c)$ in each parameter represent significant $(p<0.05)$.
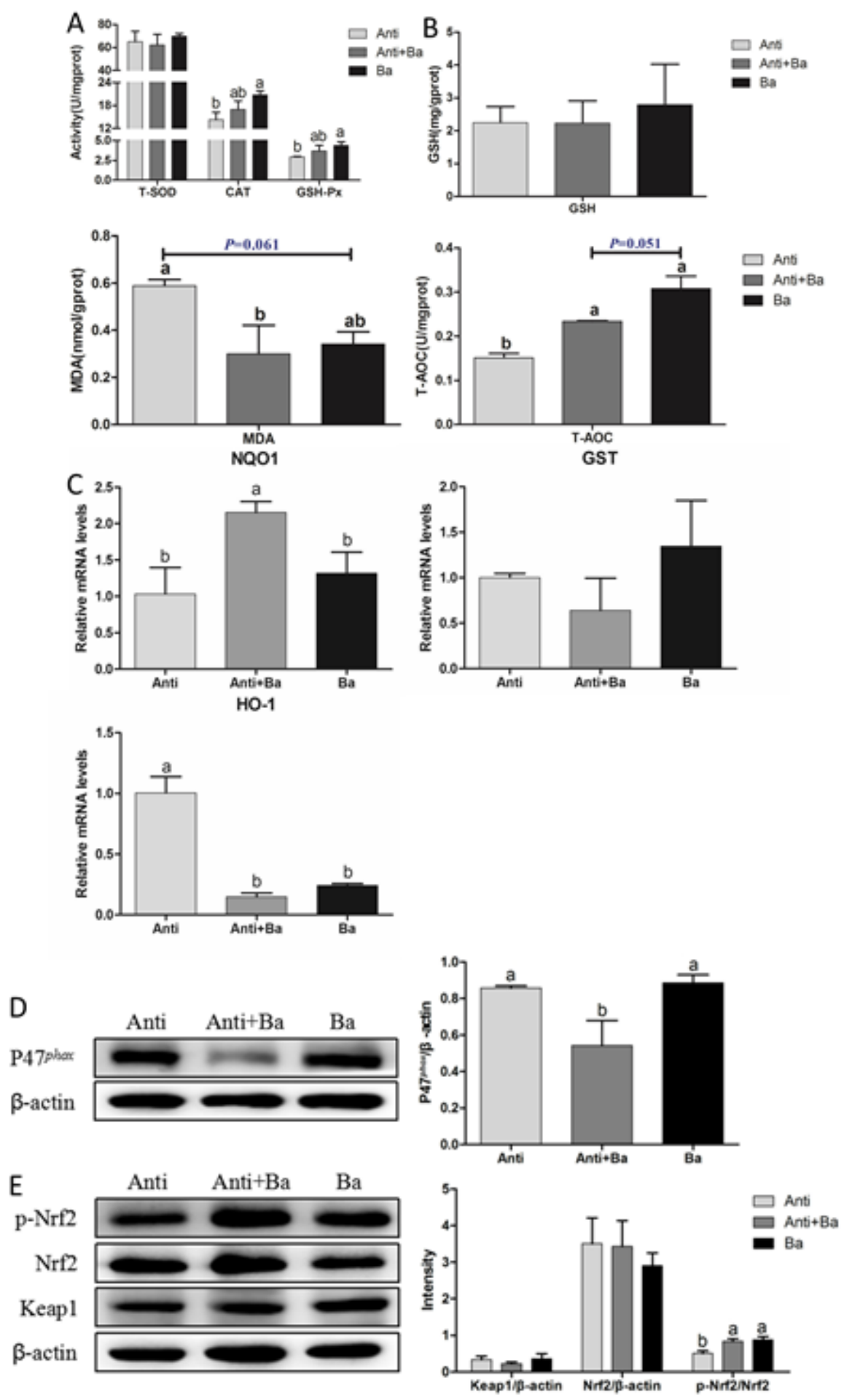


\section{Figure 3}

BaSC06 enhanced antioxidant properties and Nrf2/Keap1 signaling pathway in fattening pigs jejunum. (A囚B) The antioxidant activity was determined by measuring GSH-Px, T-SOD, CAT, GSH, MDA and T-AOC. Data are presented as the mean $\pm S D(n=8)$, one-way ANOVA with Tukey test. (C)The mRNA levels expression of genes related to anti-oxidation in jejunum of fattening pigs were analysis with RT-qPCR, the 2- $\Delta \Delta C t$ method was used to estimate mRNA abundance. (D\&E) Protein lysates from jejunal mucosa were examined by western blot for P47phox, p-Nrf2, Nrf2, Keap1 levels. The ratio of P47phox, p-Nrf2, Nrf2, Keap 1 to $\beta$-actin were analyzed using ImageJ. Different letters $(a, b, c)$ in each parameter represent significant $(p<0.05)$. 
A

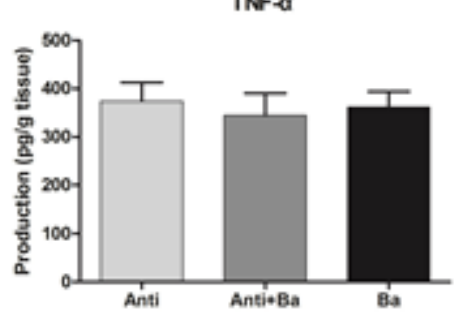

IL-6

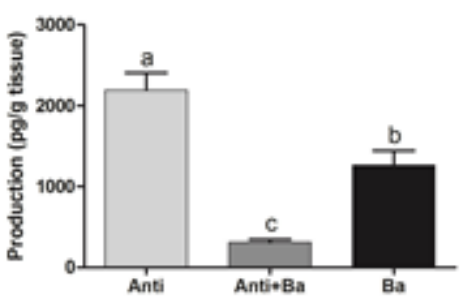

IL-8
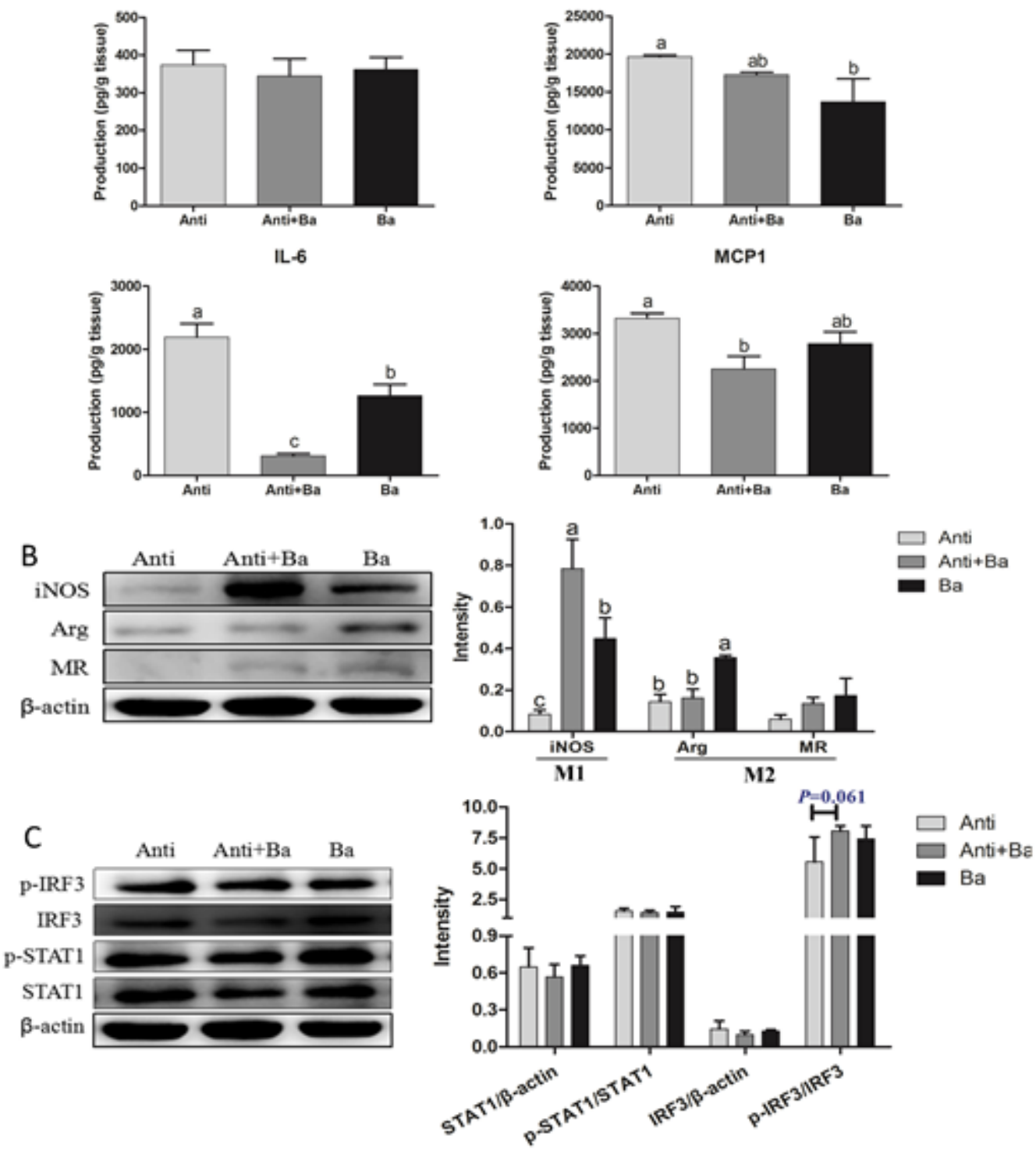

D

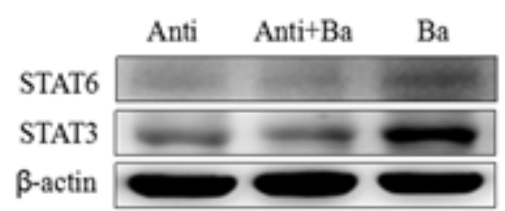

\section{Figure 4}

Effects of BaSC06 on Immune Function. (A)The Production of Immune Cytokines in ileum of fattening pigs was determined by measuring TNF-a, IL-8, IL-6 and MCP1 using ELISA. Data are presented as the mean $\pm S D(n=8)$, one-way ANOVA with Tukey test. Different letters $(a, b, c)$ in each parameter represent significant ( $p$ < 0.05). (B)Protein levels of macrophage polarized markers iNOS, Arg and MR, as well as key protein of polarization related signaling pathway p-IRF3, IRF3, p-STAT1, STAT1, STAT6 and STAT3 
were analyzed using western blotting. The ratio of iNOS, Arg, MR, STAT1, IRF3, STAT3 and STAT6 to $\beta$ actin, p-STAT1 to STAT1 and p-IRF3 to IRF3 were analyzed using ImageJ. Different letters $(a, b, c)$ in each parameter represent significant $(p<0.05)$.

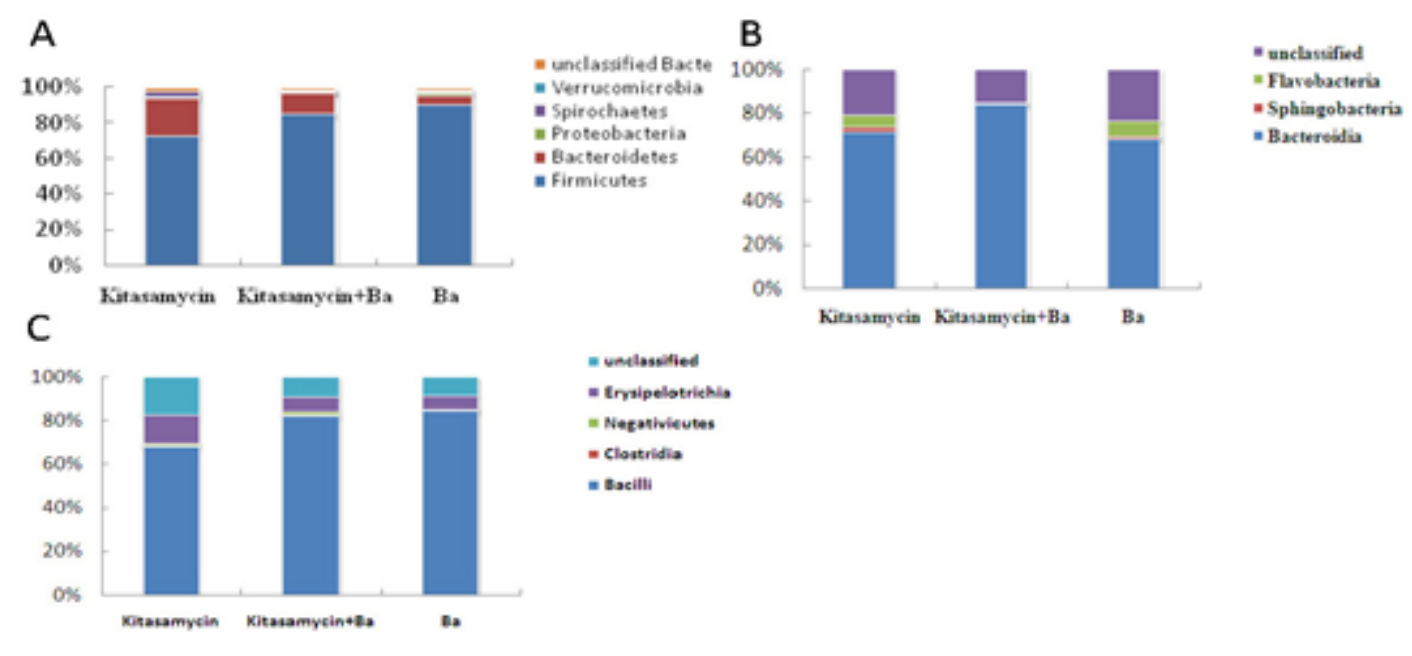

\section{Figure 5}

A BaSC06 changes the composition of intestinal microbiota without affecting the diversity of intestinal microbiota of fattening pigs. (A)Phylum classification statistical chart of flora in cecum contents of fattening pigs. (B)Phylum classification statistical chart of Bacteroidetes in cecum contents of fattening pigs. (C) Phylum classification statistical chart of Firmicutes in cecum contents of fattening pigs. 